Article

\title{
Navigation of an Autonomous Tractor for a Row-Type Tree Plantation Using a Laser Range Finder-Development of a Point-to-Go Algorithm
}

\section{Pawin Thanpattranon ${ }^{1}$, Tofael Ahamed ${ }^{2}$ and Tomohiro Takigawa ${ }^{2, *}$}

1 Graduate School of Life and Environmental Sciences, University of Tsukuba, 1-1-1 Tennodai, Tsukuba, Ibaraki 305-8572, Japan, E-Mail: fengpwt@ku.ac.th

2 Faculty of Life and Environmental Sciences, University of Tsukuba, Japan 1-1-1 Tennodai, Tsukuba, Ibaraki 305-8572, Japan, E-Mail: tofael.ahamed.gp@u.tsukuba.ac.jp

* Author to whom correspondence should be addressed;

E-Mail: takigawa.tomohiro.ff@u.tsukuba.ac.jp; Tel.: 81-29-853-4643; Fax: 81-29-853-4922.

Academic Editor: Huosheng $\mathrm{Hu}$

Received: 30 July 2015/Accepted: 31 August 2015 / Published: 7 September 2015

\begin{abstract}
It is challenging to develop a control algorithm that uses only one sensor to guide an autonomous vehicle. The objective of this research was to develop a control algorithm with a single sensor for an autonomous agricultural vehicle that could identify landmarks in the row-type plantation environment and navigate a vehicle to a point-to-go target location through the plantation. To enable such a navigation system for the plantation system, a laser range finder (LRF) was used as a single sensor to detect objects and navigate a full-size autonomous agricultural tractor. The LRF was used to control the tractor as it followed a path, and landmarks were detected "on-the-go" in real time. The landmarks were selected based on data for their distances calculated by comparison with the surrounding objects. Once the landmarks were selected, a target point was calculated from the landmarks, and the tractor was navigated toward the target. Navigation experiments were successfully conducted on the selected paths without colliding with the surrounding objects. A real time kinematic global positioning system (RTK GPS) was used to compare the positioning between the autonomous control and manual control. The results of this study showed that this control system could navigate the autonomous tractor to follow the paths, and the vehicle position differed from the manually driven paths by $0.264,0.370$ and $0.542 \mathrm{~m}$ for the wide, tight, and U-turn paths, respectively, with directional accuracies of $3.139^{\circ}, 4.394^{\circ}$, and $5.217^{\circ}$, respectively, which are satisfactory for the autonomous operation of tractors on
\end{abstract}


rubber or palm plantations. Therefore, this laser-based landmark detection and navigation system can be adapted to an autonomous navigation system to reduce the vehicle`s sensor cost and improve the accuracy of the positioning.

Keywords: control algorithm; landmarks; point-to-go; positioning; and navigation paths

\section{Introduction}

Due to the rise in population, the demand and consumption for foods have increased significantly. This creates inevitable problems of labor shortages in agricultural production. The implementation of agricultural robotics in outdoor environments can contribute to the ease of operation and reduce human drudgery. Many studies have been performed to develop sensors and control algorithms to solve the problem of the navigation of agricultural vehicles. Research on developing automatic autonomous machines for row-type plantations such as those for palm oil and rubber has attracted significant interest in the last 10 years throughout Asia. As the plantations are normally divided into plots that are not arranged in regular shapes, travelling from plot to plot is also needed for continuous operation (Figure 1). Moreover, as the tree plantations are in rows, the navigation system should navigate the vehicle to move along the path, making U-turns at the headland (Figure 2).

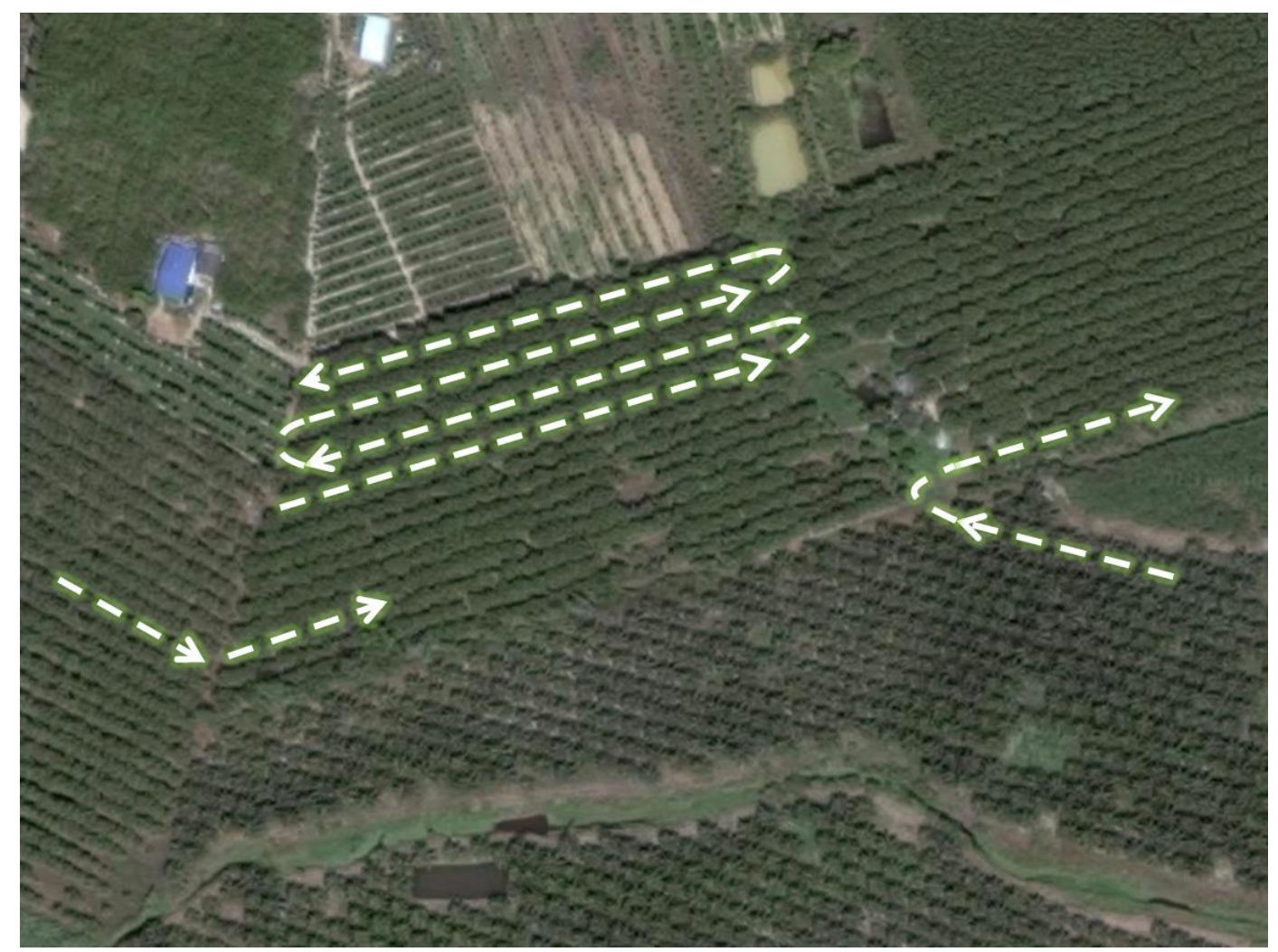

Figure 1. Various arrangements of plantations in Bo Thong, Chonburi Province, Thailand. (Google Maps, 2015). 


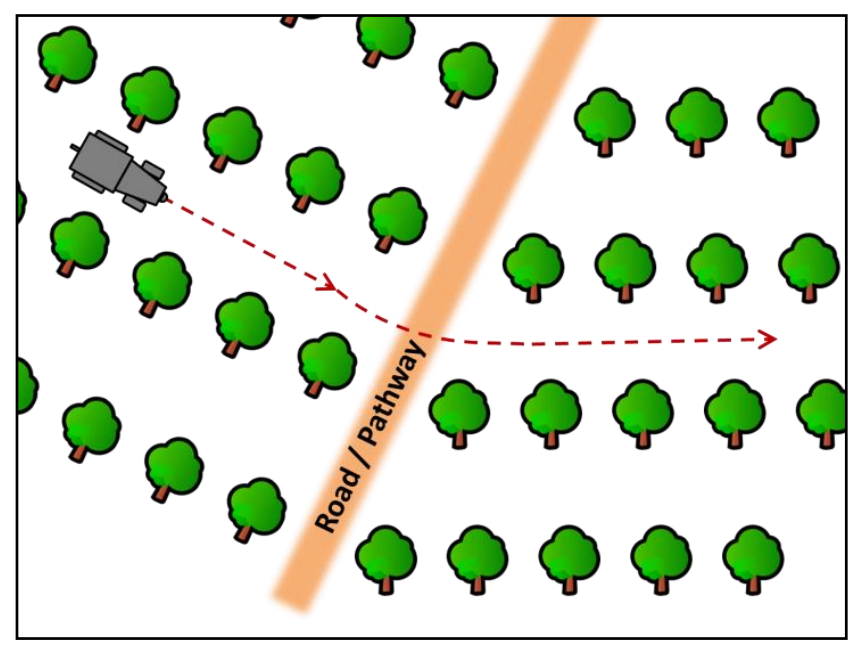

(a)

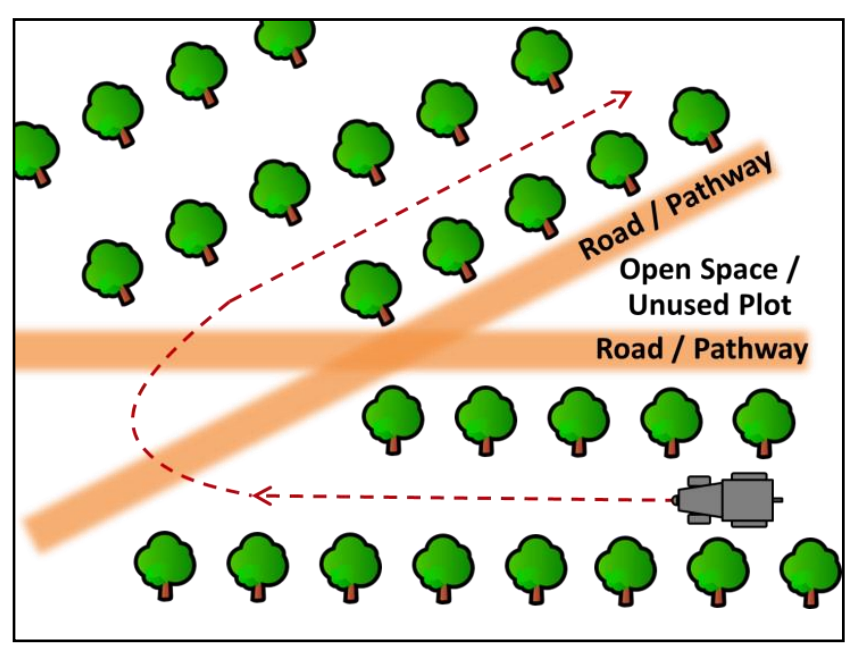

(b)

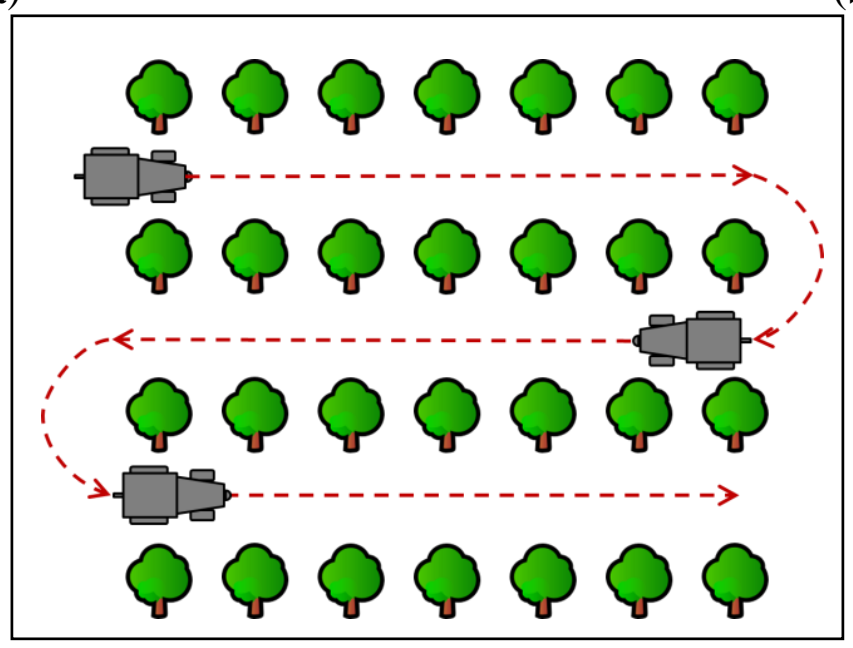

(c)

Figure 2. Tractor moving across plantation (a) traveling with a wide-curve trajectory; (b) traveling with a tight-curve trajectory and (c) U-Turn movement of tractor.

In the last decade, various navigation systems have been introduced, including dead reckoning, machine vision, and GPS systems. However, there are still difficulties with outdoor navigation due to sensor reliability. Cumulative errors in dead-reckoning or path-following navigation methods could also be reduced by real-time calculations. Light conditions introduce problems to machine vision-based control methods, but their performance is significantly improved for indoor applications. GPS systems have become popular for determining vehicle positions and contribute significantly to automatic steering in agricultural vehicles, especially in the USA and Europe, where there are large fields. A number of studies used the GPS as a base sensor for the navigation of agricultural tractors [1-5]. A GPS-based rice transplanter was developed, but it was limited by tree canopies and high-rise buildings that interfered with the signals [6]. To improve the GPS-based applications, studies related to signal acquisition in steel structures or buildings were established [7,8]. As a use of GPS for better higher accuracy, a setup for roof antenna and base station were required to use with a local positioning system and monitored structures movement [9]. Limitations on positioning measurements were resolved by laser range finders, which are suitable for outdoor applications [10,11]. 
Laser sensors are very suitable for long-range use with artificial landmarks [12,13]. Outdoors, laser rangefinders can detect landmarks with high accuracy and navigate tractors to their target positions $[14,15]$. A laser-based guidance system was capable of navigating an autonomous vehicle travelling between rows of trees in real time. The study focused solely on the recognition of trees in straight rows using a laser scanner as a navigation sensor and Hough's transform for lining up the tree rows and generating straight paths $[16,17]$. A method of control by finding the midpoint of a path constructed by $90 \mathrm{~cm}$ high hay bales as walls on both sides of the path was also reported [18]. The autonomous navigation of a combine harvester by detection of the crop height using LRF has been studied [19]. A map-based method was reported that enables the development of an unmanned ground vehicle using an LRF and a map of a test field [20]. However, the map-based method requires maps to be surveyed in advance for the navigation system to work.

There are many opportunities to implement laser sensors in autonomous systems due to their cost, reliability and advantages compared to other sensors, as it can be used in different lighting conditions and under tree canopies. The above research focused on straight runs or approaches to target positions using landmarks. The number of landmarks was limited, or reflectors were used at the target position. However, for navigation under a tree canopy, continuous landmark detection is important for navigating a vehicle. An autonomous control system was developed using a laser range finder as a basic sensor to recognize artificial landmarks [21,22]. Target points were calculated using a two nearest landmark selection method. Landmark selection is very important for finding the positions of agricultural vehicles in places where GPS signals are interrupted. The point-to-go algorithm has the potential for navigation under these conditions using an LRF. In addition, the use of fewer sensors reduced the complexity of the algorithm and the cost of the control system. A single sensor navigation system with satisfactory performance would facilitate the use of autonomous systems in agricultural operations.

The objective of this research was to develop an LRF-based navigation system that could identify landmarks in the environment and navigate a vehicle to a point-to-go target location, especially in a row-type plantation.

\section{Materials and Methods}

\subsection{Instrument Setup}

A Kubota Kingwel tractor (KL-21, 15.4 kW, Kubota, Osaka, Japan) was used for the experiments. The full-size tractor was modified with an autonomous control unit using a hydraulic actuator for steering, shifting gears and braking. A programmable logic control (PLC), Keyence KZ-A500 (Keyence, Osaka, Japan), with digital input/output, analog input/output and encoder pulse encounter PCI-cards, was installed for signal communication between the computer unit and the hydraulic actuator. A laser range finder (LRF), SICK LMS551-Lite (Sick, Waldkirch, Germany), was used in the front of the tractor, $35 \mathrm{~cm}$ above ground level (Figure 3). This LRF had an $80 \mathrm{~m}$ maximum scanning range with a $190^{\circ}$ scanning angle. The angle resolution was set at $0.25^{\circ}$ with a $25 \mathrm{~Hz}$ response rate. A GPS system, Trimble MS750 (Sunnyvale, CA, USA) was set up for collecting the tractor position during experiments under RTK-GPS operation mode with an accuracy of $\pm 2 \mathrm{~cm}$. 


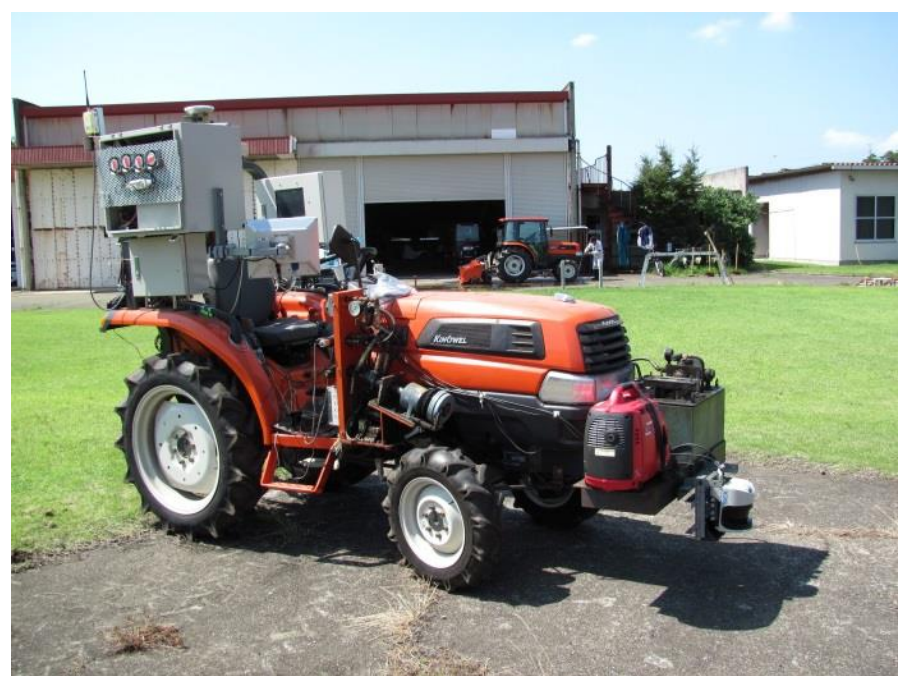

(a)

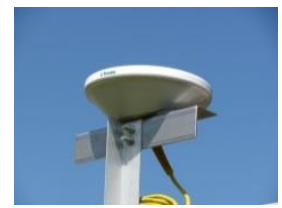

(c)

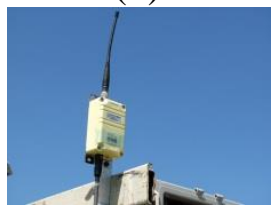

(d)

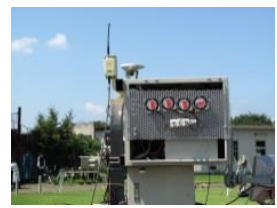

(e)

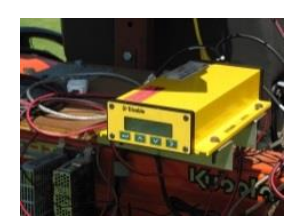

(b)

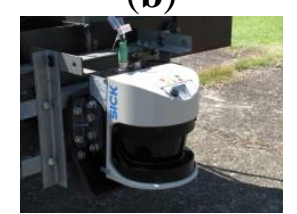

(f)

Figure 3. Experimental tractor and its components (a) Test Tractor, Kubota Kingwel KL-21; (b) GPS Receiver, Trimble MS750; (c) GPS Antenna; (d) Radio Transmitter for RTK-GPS operation mode; (e) Computer and PLC Control Unit, Keyence KZ-A500; and (f) Laser Range Finder, SICK LMS551.

\subsection{Navigation Control Algorithm}

The navigation control algorithm was developed with four steps. First, all of the objects within the range of the LRF were collected. Initially, the data consisted of different objects including landmarks. Second, the objects were categorized into two types: landmarks and non-landmark objects. Third, the landmark objects were filtered and considered for tractor navigation, and a centroid of an area covered by landmarks was determined as a target to run the tractor in the forward direction. Finally, the steering angle was calculated, and the signal was sent to the hydraulic actuator to navigate the tractor to the target point. The details of each procedure are discussed in Sections 2.2.1-2.2.5.

All four steps were conducted continuously until the tractor ran to the end of the path and stopped. The tractor was stopped autonomously when landmarks were detected within the safety range of $1 \mathrm{~m}$. The control loop of this navigation system required 500-600 ms for each cycle (Figure 4). 


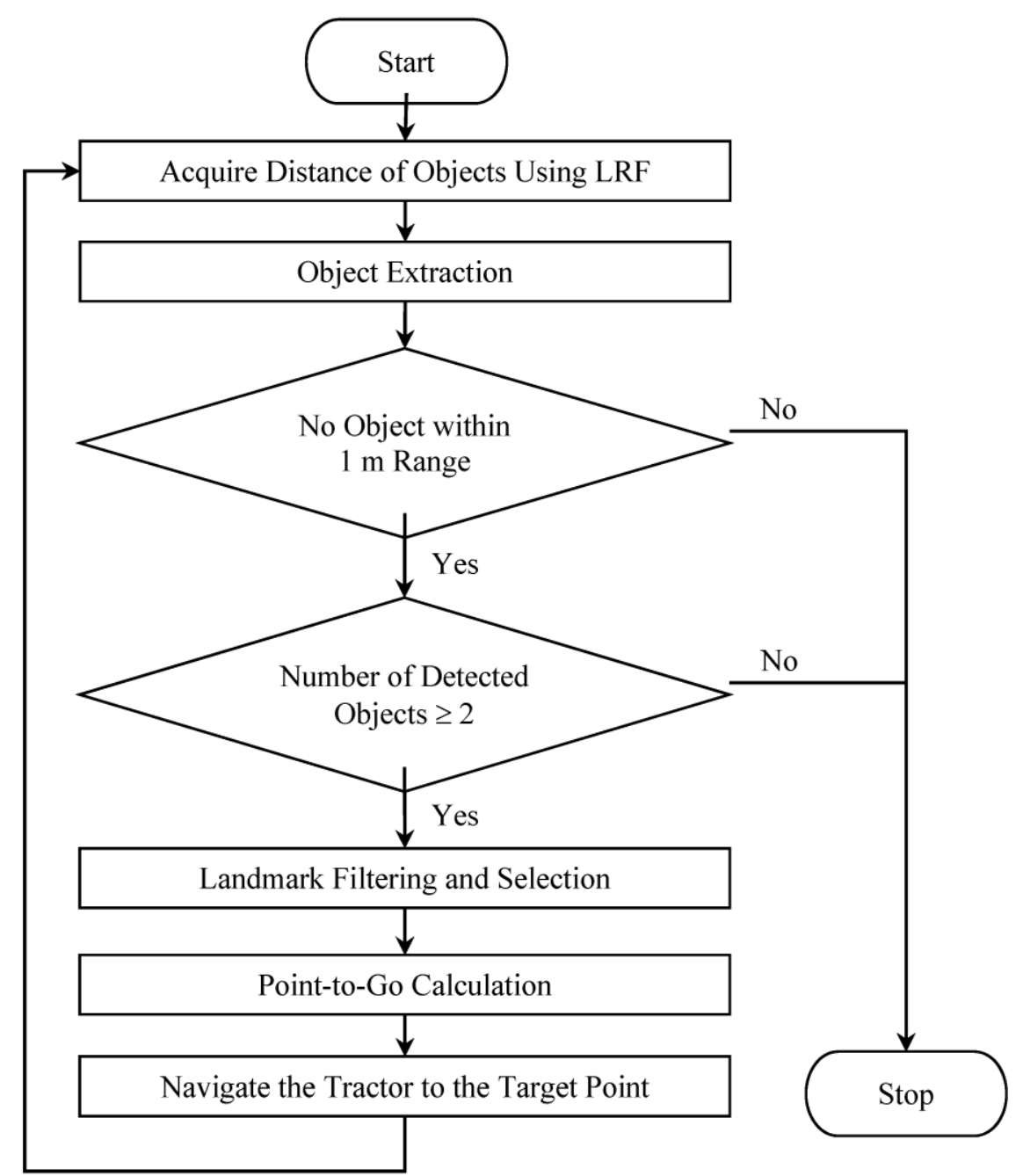

Figure 4. Navigation system using landmarks.

\subsubsection{Object Extraction}

In the algorithm, the extracted objects were clustered according to nearest-neighbor distances. The object pattern was identified from the LRF data, and the distances of the objects were obtained. The SICK LMS551-lite LRF was set at a $190^{\circ}$ scanning angle with a $0.25^{\circ}$ angular resolution. In each scan cycle, 760 data sets were received.

The data were clustered based on the threshold value $(\delta)$ determined from a flat panel (Figure 5). The flat panel was placed at a distance (d) from the LRF with an inclination $(\theta)$ to the perpendicular axis of the LRF. The distances were recorded as $d_{i}$ and $d_{i+1}$ at scan steps $i$ and $i+1$, respectively. The threshold value can be calculated from the difference between two adjacent points of an object:

$$
\theta=\left|d_{i}-d_{i 1+1}\right|=\left|d_{i}-\frac{d_{i}}{\cos 0.25}-\frac{d_{i} \tan 0.25 \tan }{\cos 0.25-\sin 0.25 \tan }\right|
$$




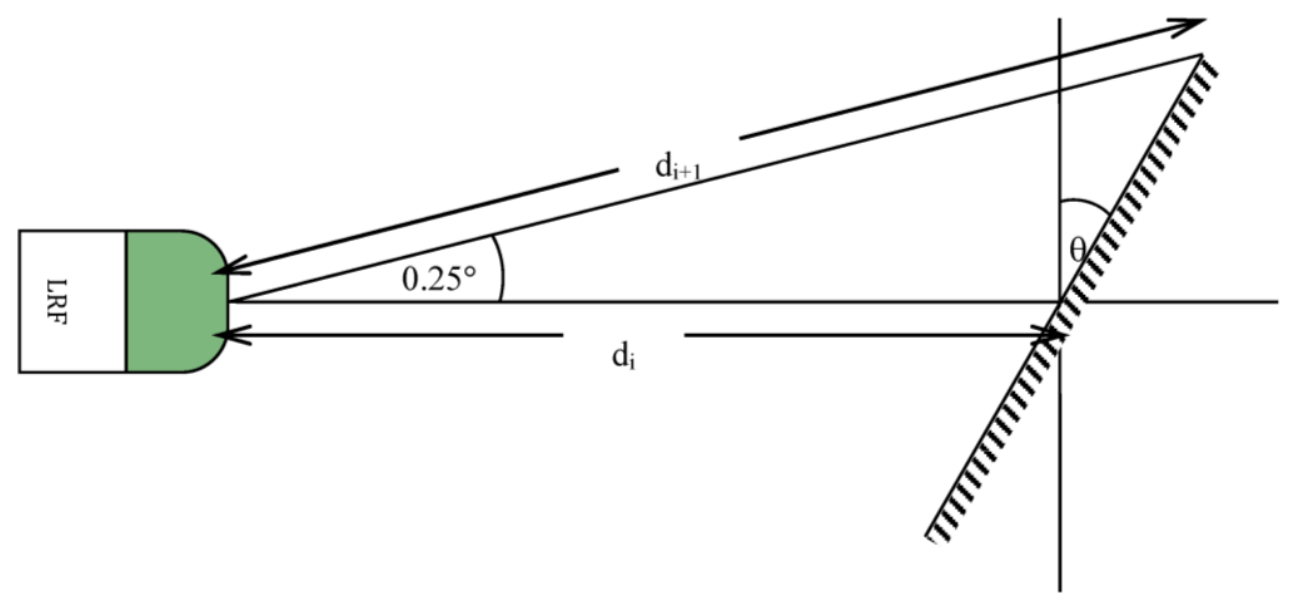

Figure 5. Geometrical relation between the LRF and the flat panel in the scanning steps of the laser range finder (LRF).

The threshold value between the points of an object was changed due to the measured distance from the LRF. The larger the distance to measure, the higher the threshold value that was observed (Figure 6).

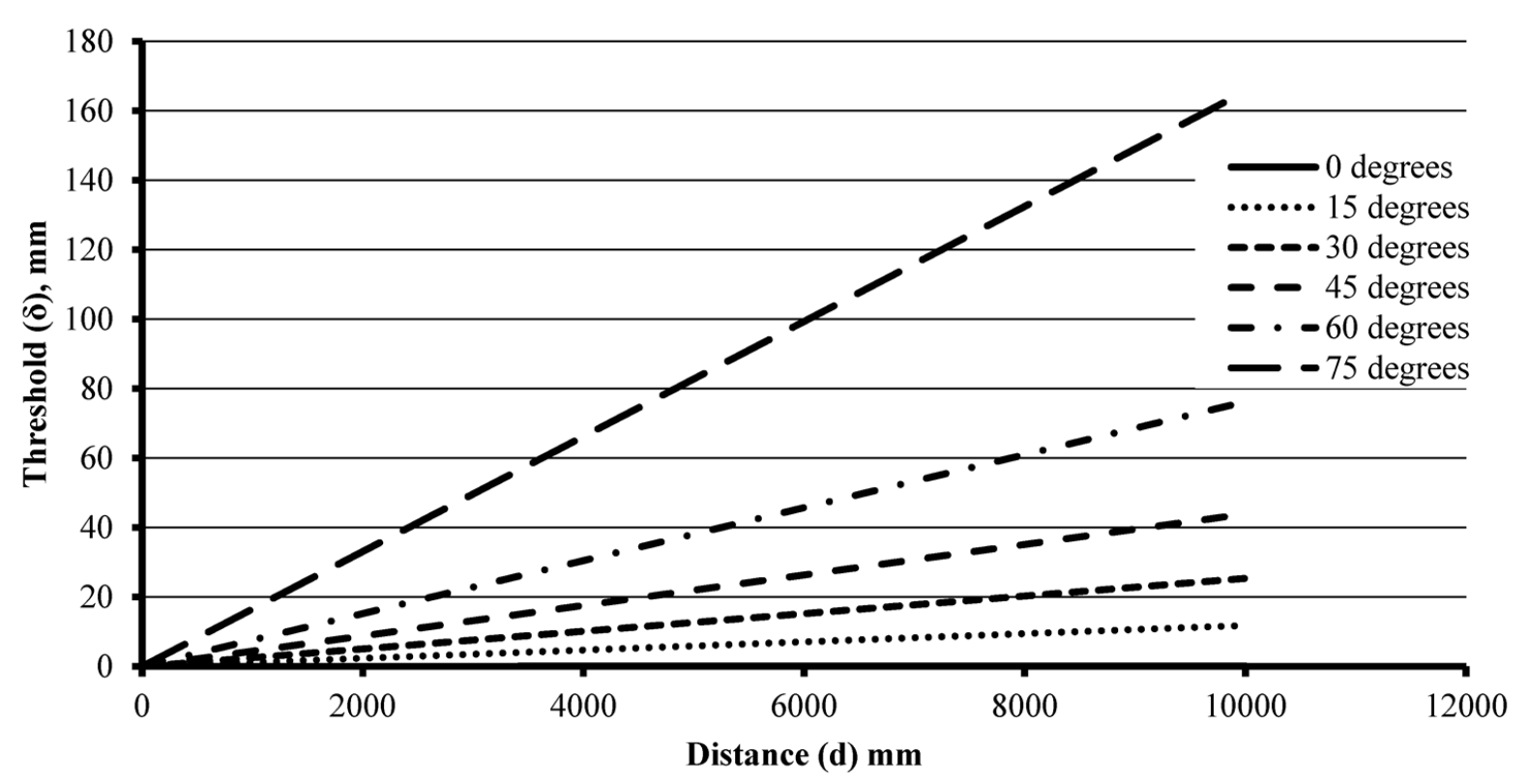

Figure 6. Threshold value $(\delta)$ at different distances from the LRF at different inclinations $(\theta)$.

The amount of data $(n)$ from scanning the LRF depends on the diameter of the object $(D)$ and the distance of the object $(d)$ from the LRF (Figure 7). The amount of data ( $n$ ) can be expressed as

$$
n \approx\left[2 \times \operatorname{int}\left(\frac{D}{2 d \tan 0.25}\right)\right]+1
$$




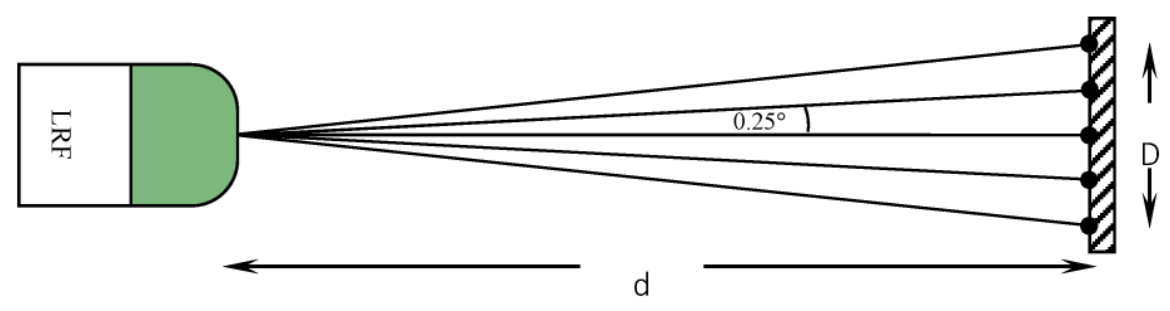

Figure 7. Scanning profile with the lowest scanning resolution for an object at a given distance away

Three objects of different sizes $(100,150$ and $200 \mathrm{~mm})$ were examined to determine the number of data that could be received when the objects were placed at different distances from the LRF (Figure 8).

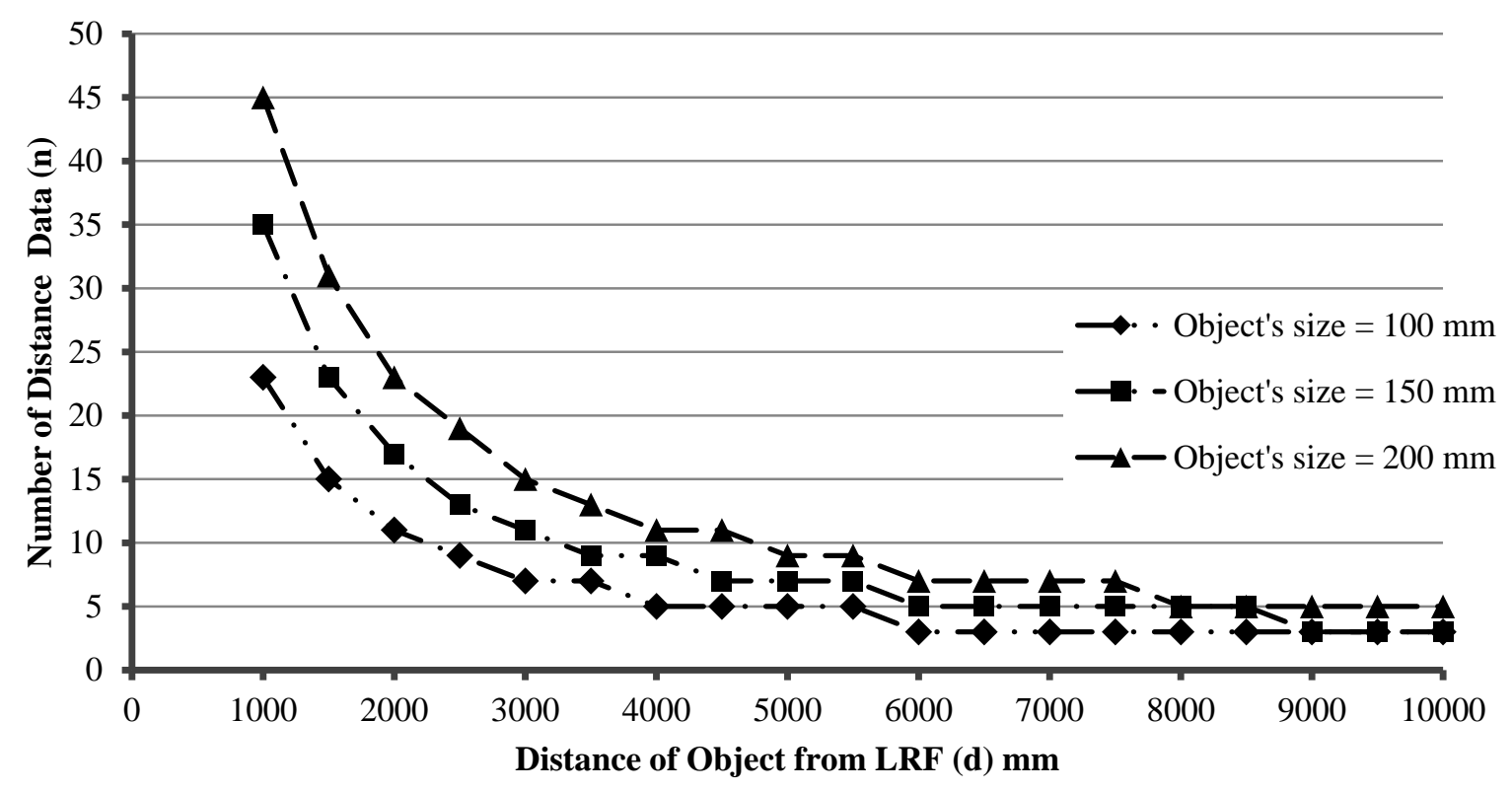

Figure 8. Data for different-sized objects at different distances from the LRF.

The landmarks used in this research were traffic cones of $70 \mathrm{~cm}$ height and $40 \mathrm{~cm}$ base width. Because of the height and the conical shape of the landmarks, the LRF was installed $35 \mathrm{~cm}$ above ground level at the front of the tractor (Figure 3f). The cone diameter at the level that the LRF detected was $16 \mathrm{~cm}$. The number of data $(n)$ for each of the landmarks at a range of $6 \mathrm{~m}$ should not be less than 5 . The detectable diameter of the cone was $16 \mathrm{~cm}$. Therefore, the objects with diameters in the range from 12 to $20 \mathrm{~cm}$ were considered as landmarks. Each object contained parameters of the relative distance to the tractor and size. The flowchart of the Object extraction algorithm depicts the data-receiving process from the LRF and landmark selection (Figure 9). 


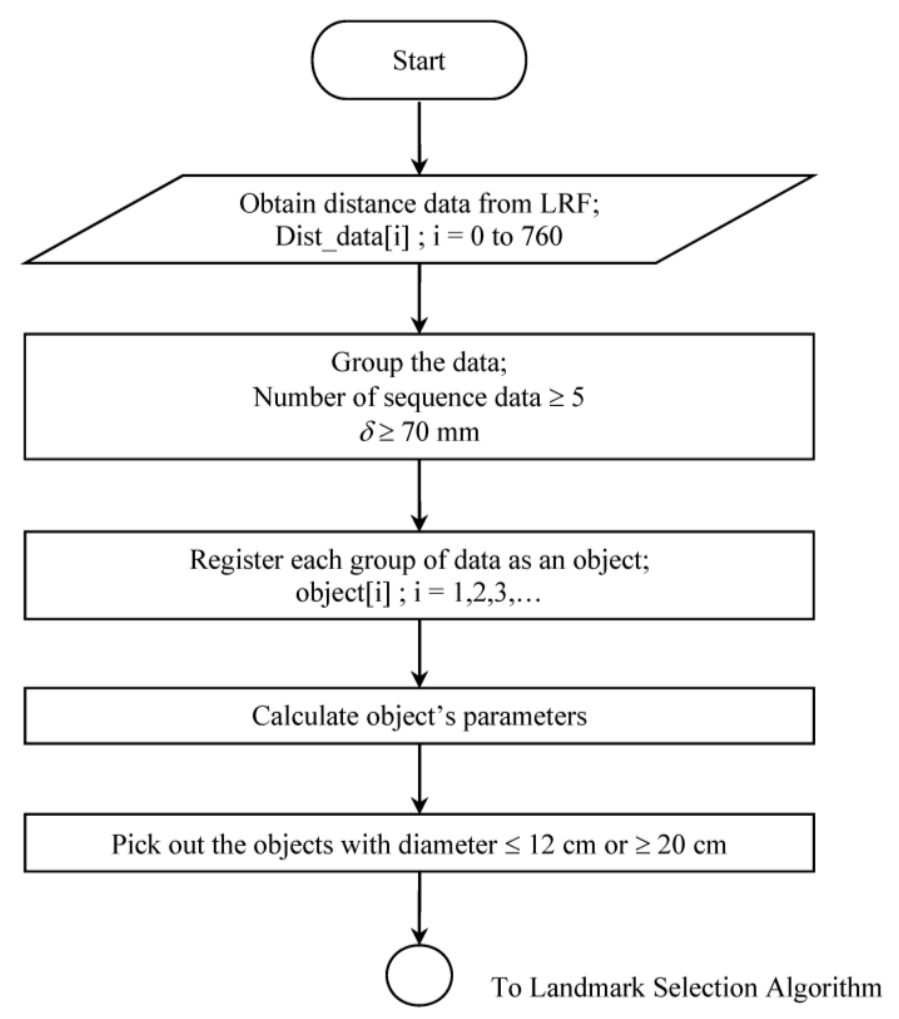

Figure 9. Object extraction algorithm.

\subsubsection{Landmark Selection from Extracted Objects}

After the objects were extracted, some of them were selected as landmarks to be used to navigate the tractor. The LRF started by finding the objects that were closest to the tractor and gradually found objects that were farther away. The objects were sorted in order of ascending distance from the LRF. At the beginning, all of the objects extracted from the LRF data were sorted by distance. If more than four objects were extracted, then the four nearest objects were selected as landmarks. When the number of objects was between two and four, the system selected all of the objects. This algorithm is referred to as the landmark selection algorithm, or as point-to-go (Figure 10).

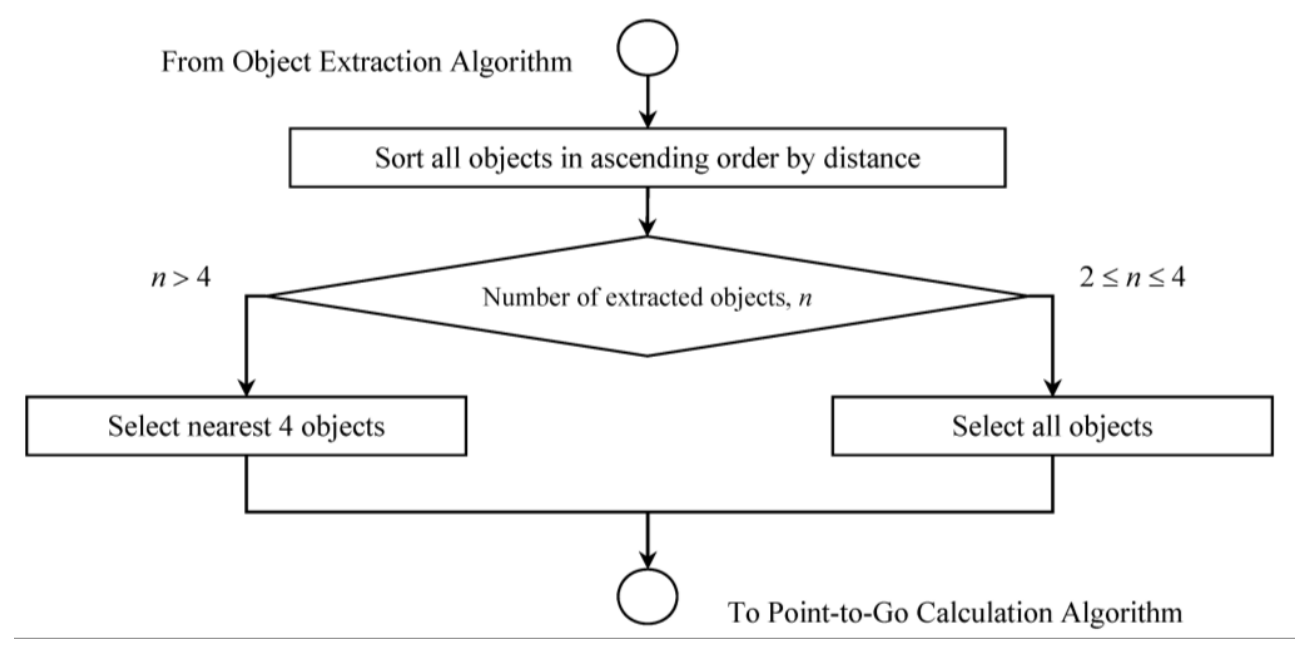

Figure 10. Landmark selection algorithm. 


\subsubsection{Landmark Selection Filtering}

To improve the efficiency of the control system, it is important to select the appropriate landmarks from the other objects in an optimal manner, a very challenging task. During the navigation, the system might detect other objects, such as other moving machines or other fieldwork operators. To set aside these types of data so as not consider them the calculation of point-to-go, a landmark selection-filtering algorithm was added. The unfiltered landmarks of the current scan cycle were compared with those of the previous one. Three parameters were noted: the relative lateral distance to the LRF, the relative longitudinal distance to the LRF, and the landmark radius. For ease of explanation, the unfiltered landmark data were projected on a layer, while the landmark data from previous scan cycle were projected on another layer. By overlapping these two layers, only the four nearest landmarks that overlapped with small differences were selected. Because the appropriate landmarks were stationary and the tractor speed was nearly constant, changes in the landmark distance data were also nearly constant. Using this principle, other moving objects and oversize or undersize objects were mostly eliminated (Figure 11).

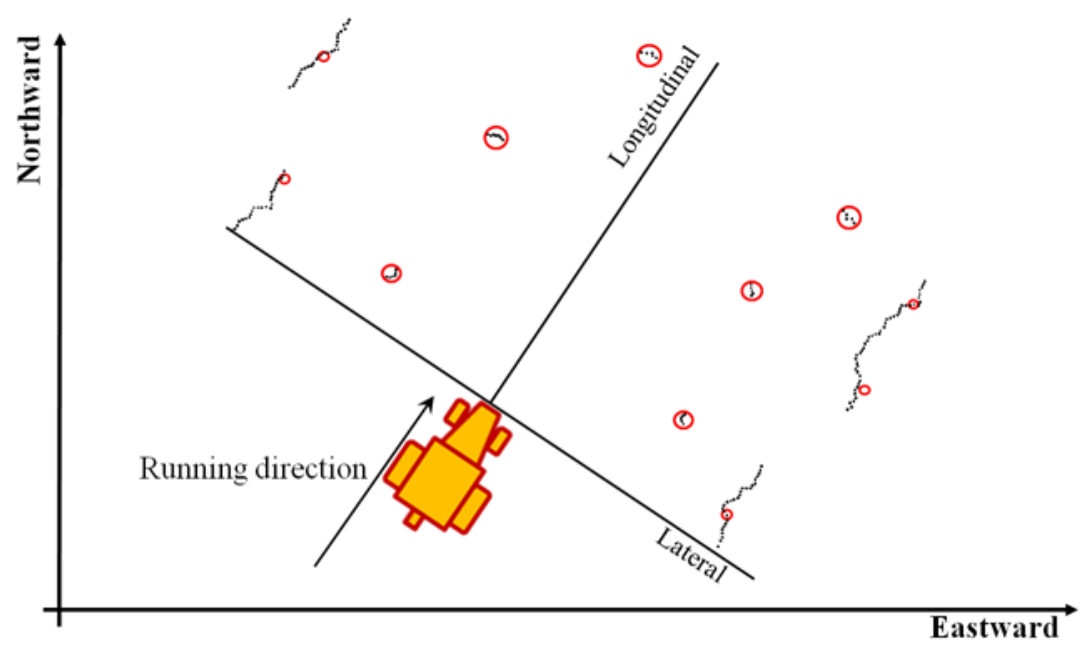

(a)

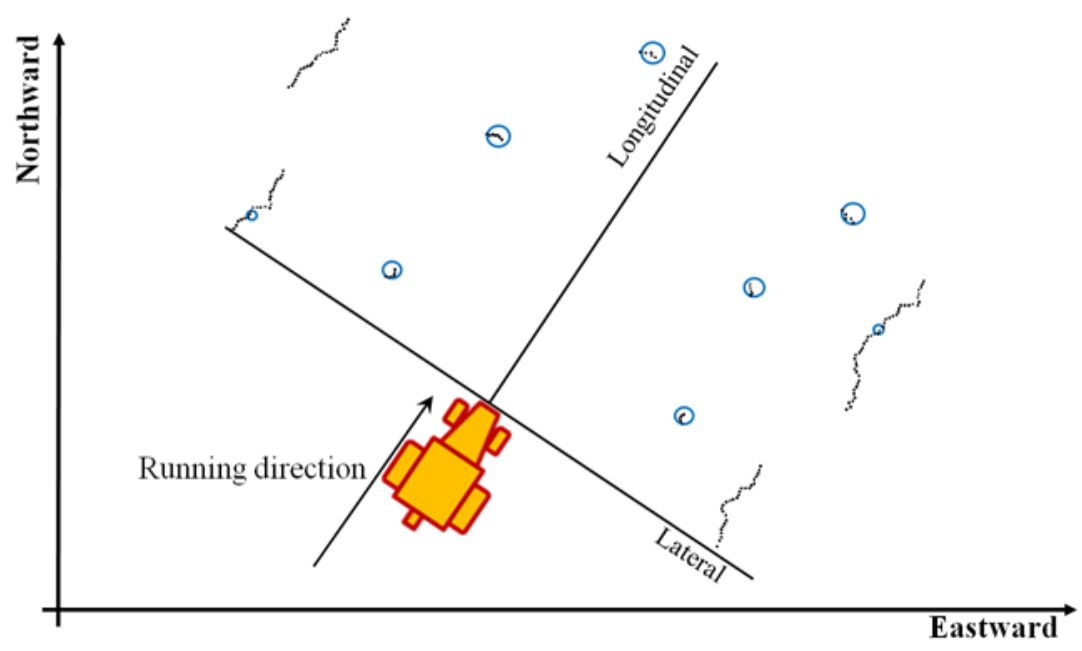

(b)

Figure 11. Cont. 


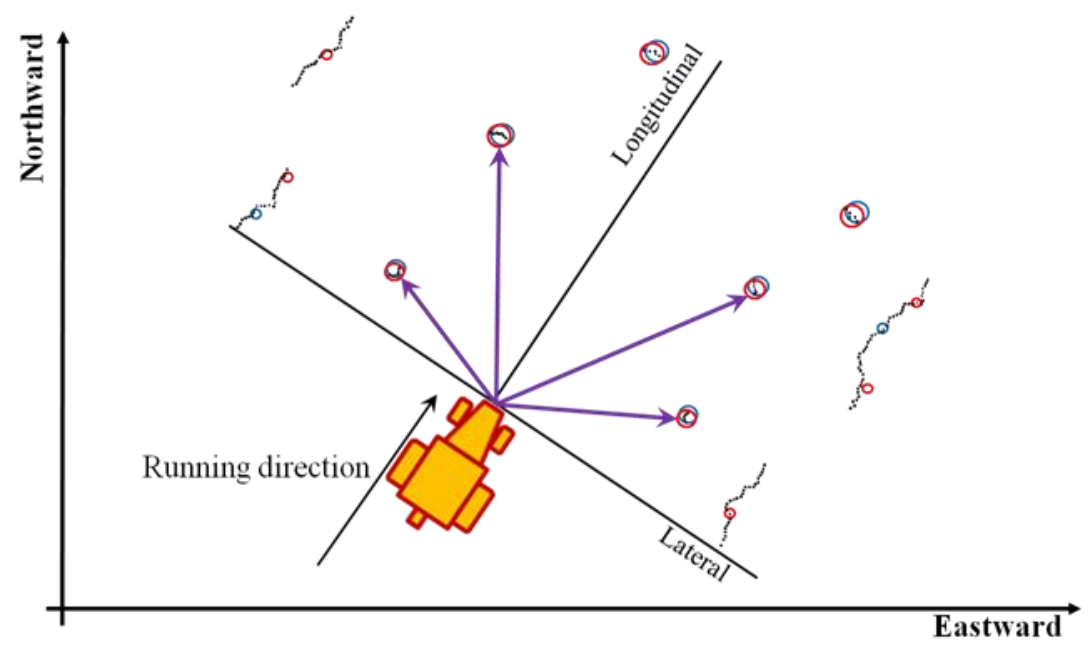

(c)

Figure 11. Layer comparison in landmark selection filtering (a) unfiltered landmarks;

(b) previous landmarks; (c) overlapping of (a) and (b).

The algorithm received unfiltered landmark data from the landmarks selection algorithm. The Point-to-Go calculation algorithm needs two to four landmarks for calculation. Four array slots of data were reserved for the filtered landmarks. Therefore, if the unfiltered landmark number was less than four, the landmark data in the empty array slot were all set to zero. If the data received were a first set of data, there were no previous landmarks to be compared at this state, and then these landmarks were used for the point-to-go calculation without filtering. Then, the data were set as previous landmark data, which were used in the next scan cycle. In case the system already recorded previous landmark data, the size and distance data of each unfiltered landmark were compared with the data of the previous landmarks. During this state, only landmarks that have differences in size and distance from the LRF that do not exceed threshold values were selected. As the experimental tractor speed was $0.18 \mathrm{~m} / \mathrm{s}$ and the data acquisition rate was $2 \mathrm{~Hz}$, the experimental tractor moved approximately $90 \mathrm{~mm}$ forward in one cycle of LRF data acquisition. During turning, the experimental tractor displacement also depended on the steering speed. The threshold value of the distance difference was set to $500 \mathrm{~mm}$. Finally, the filtered landmark data were recorded as previous landmarks for the next scan cycle filtering and for the Point-to-Go Calculation algorithm (Figure 12).

\subsubsection{Point-to-Go Calculation}

Once the landmarks were selected, a polygon area was created from their positioning information (Figure 13). Each of the landmarks had a position $\left(x_{i}, y_{i}\right)$ relative to the tractor's position $\left(x_{0}, y_{0}\right)$. The polygon area $(A)$ was calculated as

$$
A=\frac{1}{2} \sum_{i=0}^{n-1}\left(x_{i} y_{i+1}-x_{i+1} y_{i}\right)
$$

Then, the centroid of the polygon $\left(c_{x}, c_{y}\right)$ was calculated from the positions of the landmarks 


$$
\begin{aligned}
& c_{x}=\frac{1}{6 A} \sum_{i=0}^{n-1}\left(x_{i}+x_{i+1}\right)\left(x_{i} y_{i+1}-x_{i+1} y_{i}\right) \\
& c_{y}=\frac{1}{6 A} \sum_{i=0}^{n-1}\left(y_{i}+y_{i+1}\right)\left(x_{i} y_{i+1}-x_{i+1} y_{i}\right)
\end{aligned}
$$

where $\left(x_{n}, y_{n}\right)=\left(x_{0}, y_{0}\right)$.

The centroid $\left(c_{x}, c_{y}\right)$ of the polygon area (A) was selected as the point-to-go and used as the target point to navigate the tractor.

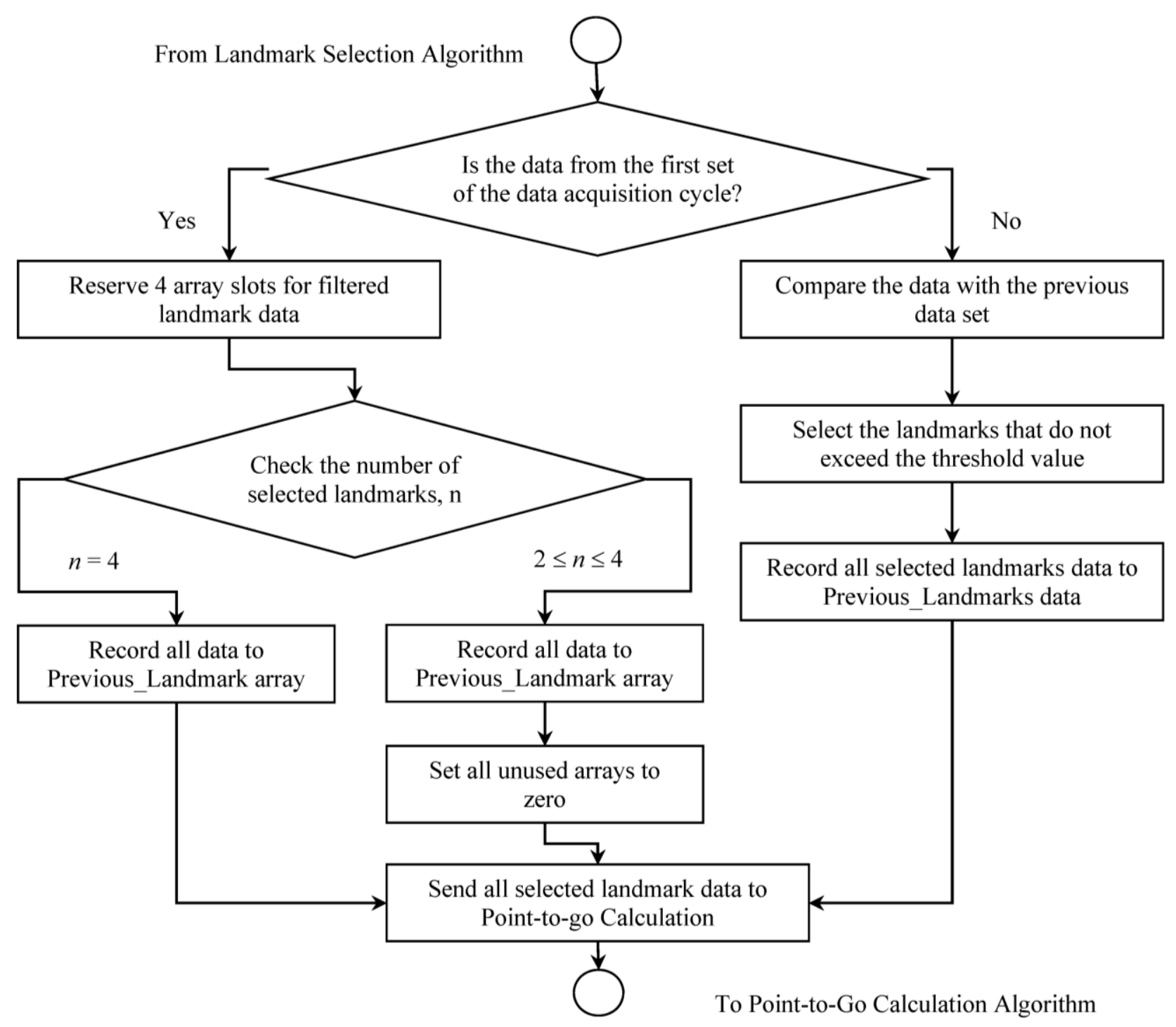

Figure 12. Landmark selection filtering algorithm. 


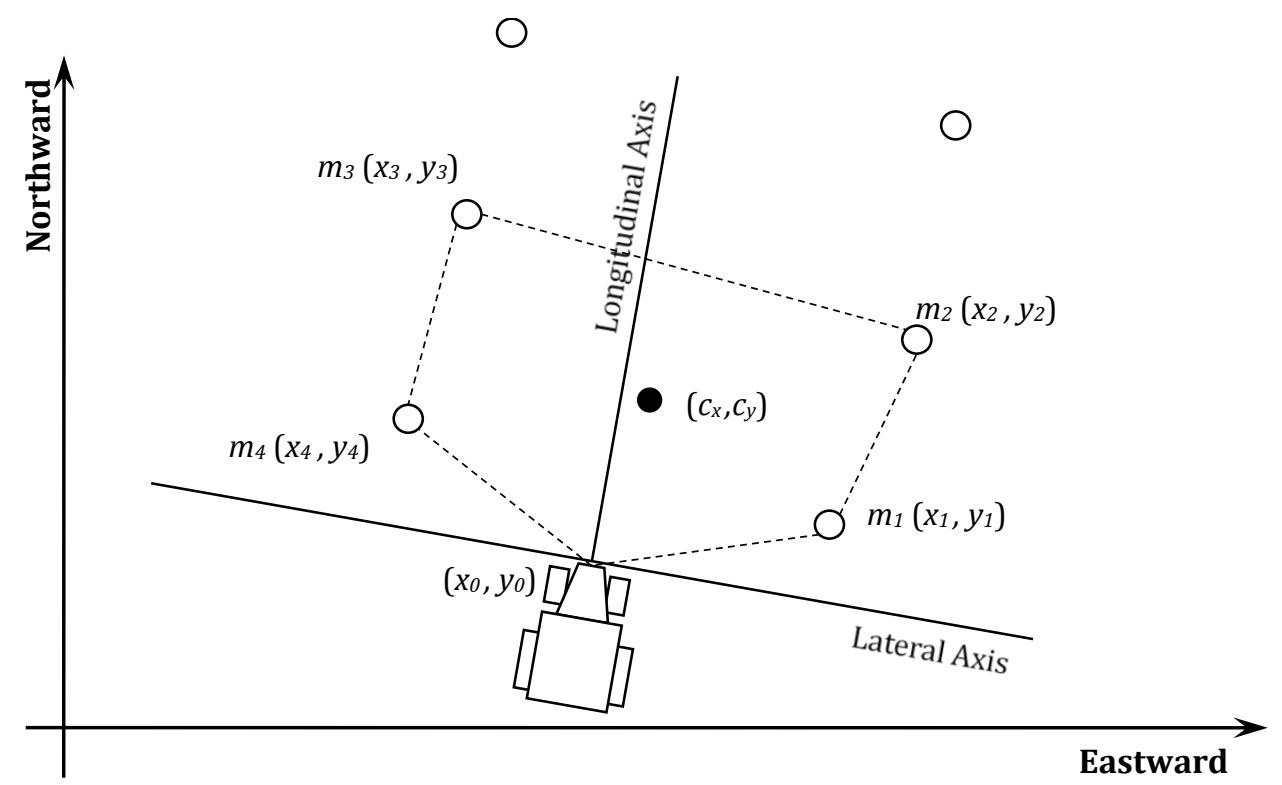

Figure 13. The centroid of the polygon formed by the landmarks.

\subsubsection{Steering Control}

Once the centroid of polygon area was determined, the centroid point was set as a target point for the navigation of the tractor. A steering angle $(\alpha)$ was calculated from the centroid point (Figure 14):

$$
\alpha=\tan ^{-1} \frac{c_{x}}{c_{y}}
$$

This steering angle value was sent to the hydraulic actuators to steer the tractor to the target position. To prevent damage to the steering controller, the steering angle was maintained between $-35^{\circ}$ and $+35^{\circ}$ $\left(0^{\circ}\right.$ for the neutral position, positive values for the counter-clockwise direction, and negative values for the clockwise direction).

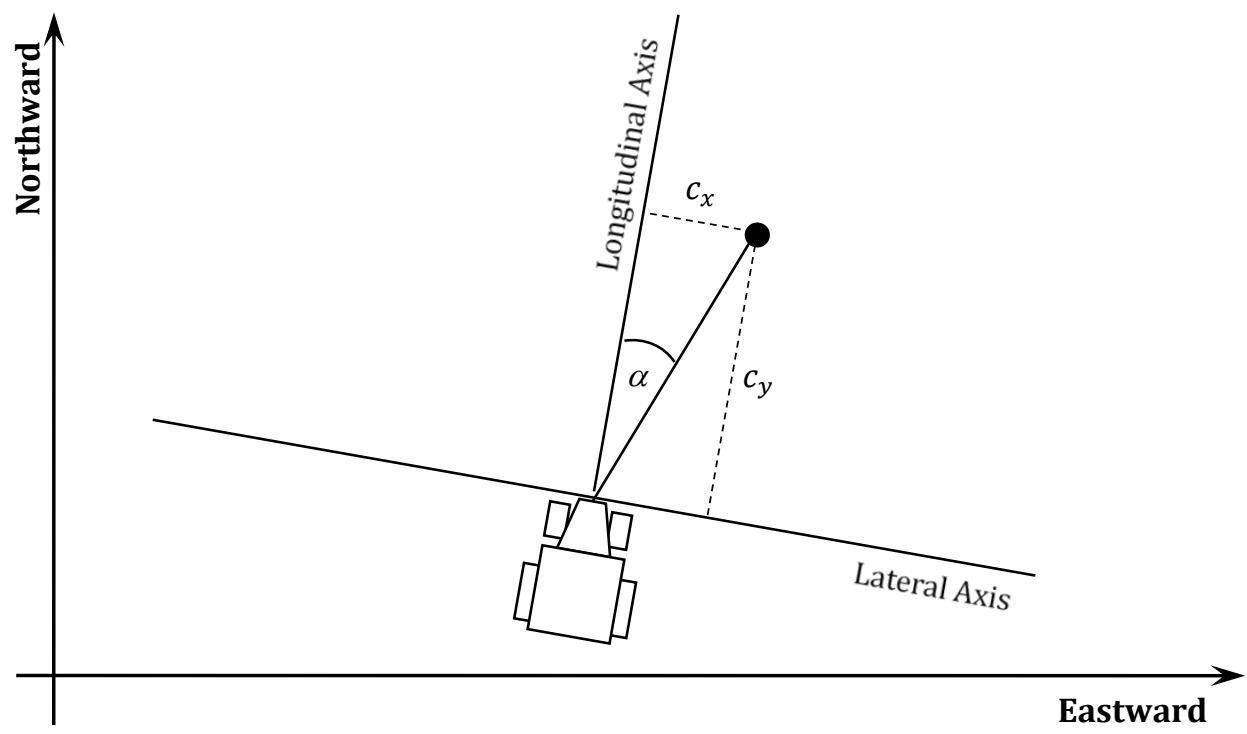

Figure 14. Calculation of the steering angle. 


\subsection{Navigation Using Landmarks}

\subsubsection{Experimental Paths}

An autonomous tractor-trailer was developed to evaluate the performance of the control system before deploying it on a real agricultural field. To focus the performance of the algorithms, experiments were performed on artificial paths constructed using traffic cones. The similarity of the LRF data obtained from cone scanning was compared with data from a para-rubber plantation in Thailand (Figure 15). The detection algorithms had the capability to detect the para-rubber trunks as well as the cones. The experiments were performed at the Agricultural and Forestry Research Center of the University of Tsukuba, Japan. Test paths were constructed on a flat concrete plot of $13 \mathrm{~m}$ by $30 \mathrm{~m}$. The cones were placed on both sides of the path, with each path being $5 \mathrm{~m}$ in width. Three different test courses, Wide curve, Tight curve and U-turn run, were used (Figure 16).
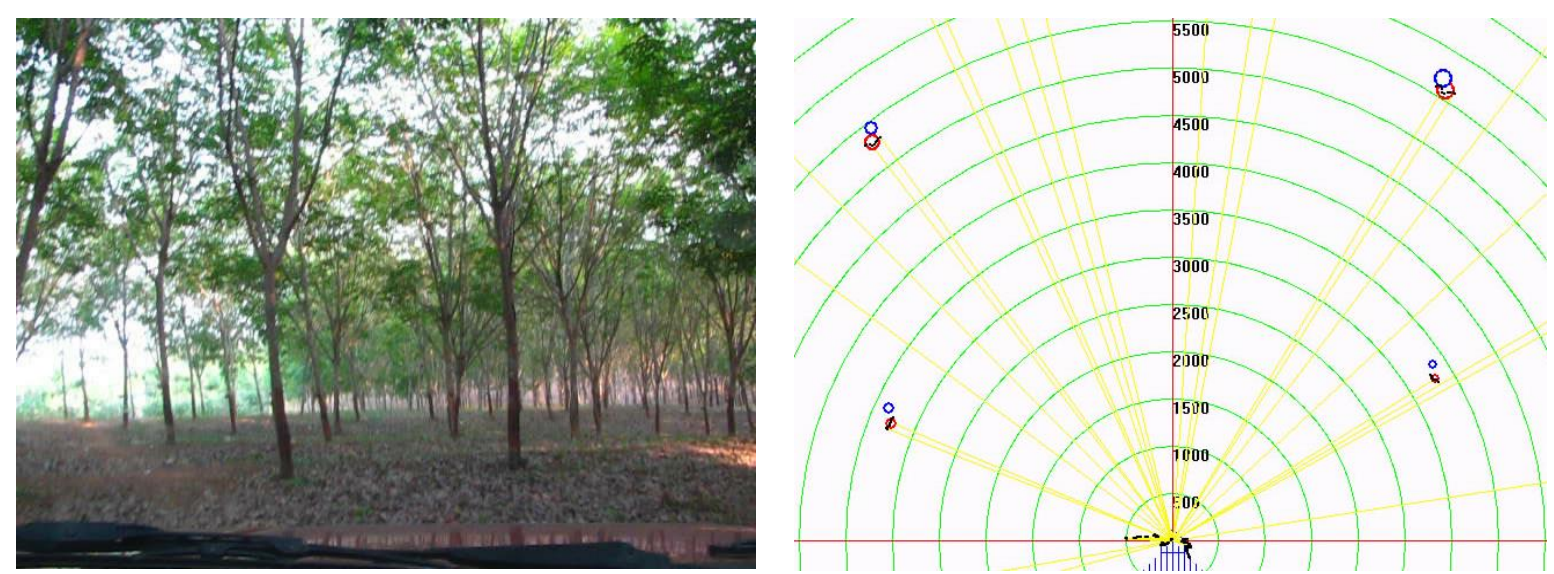

(a)
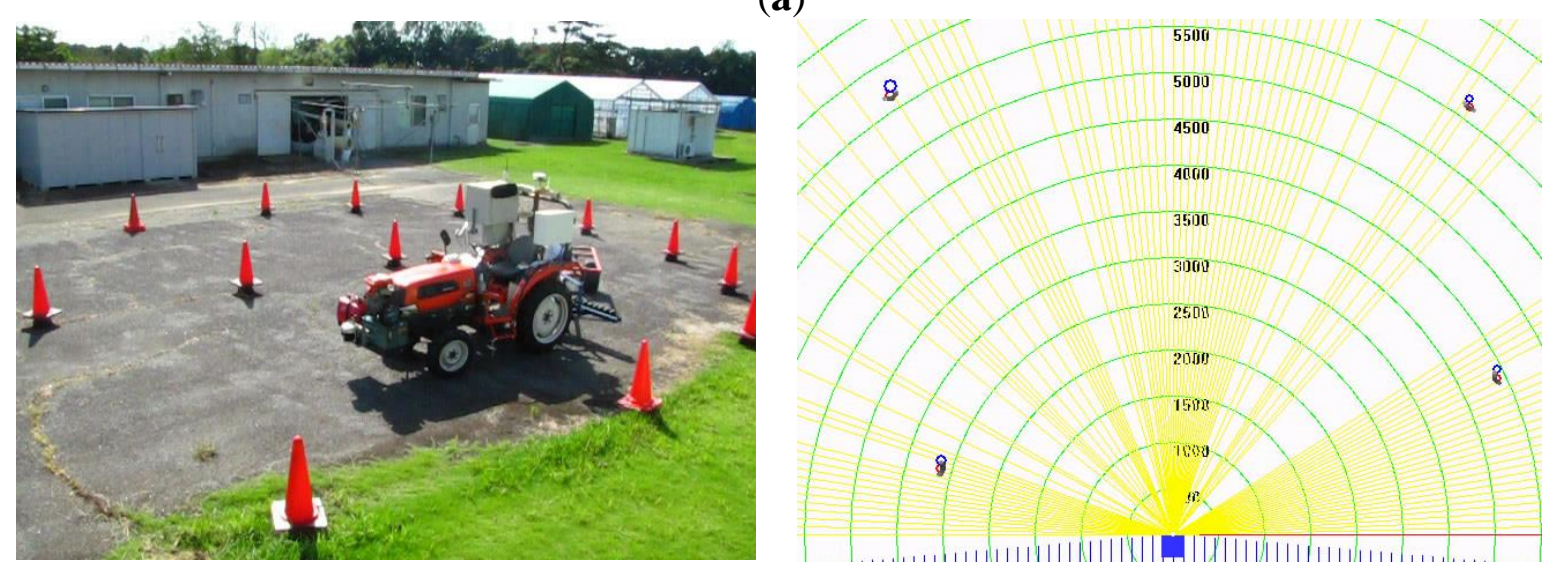

(b)

Figure 15. LRF data from (a) the para-rubber plantation and (b) the course constructed using traffic cones. 


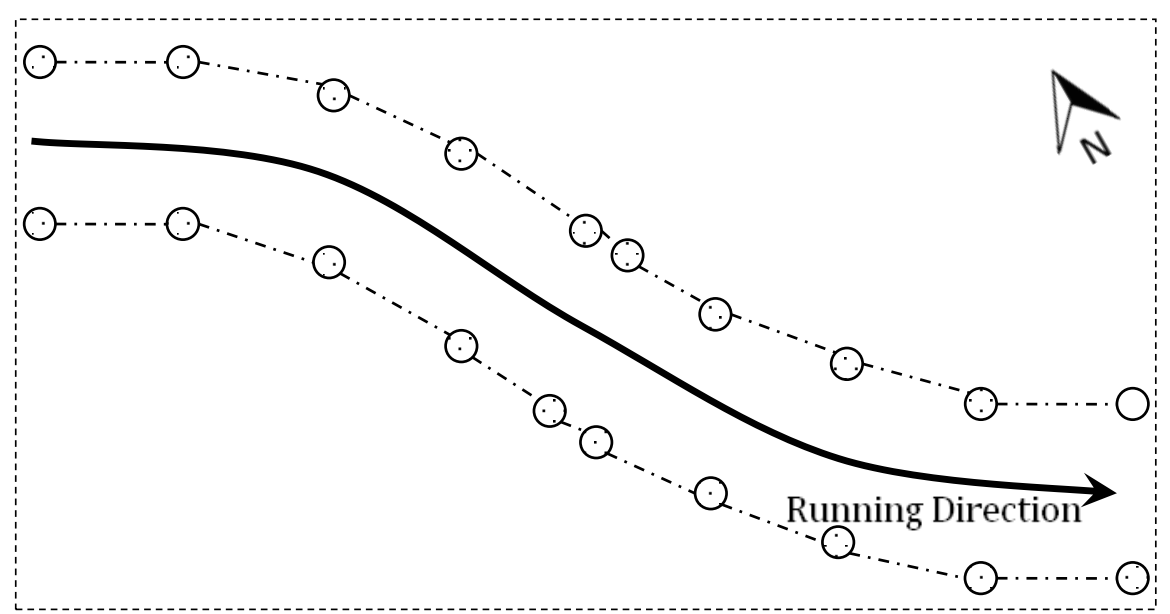

(a)

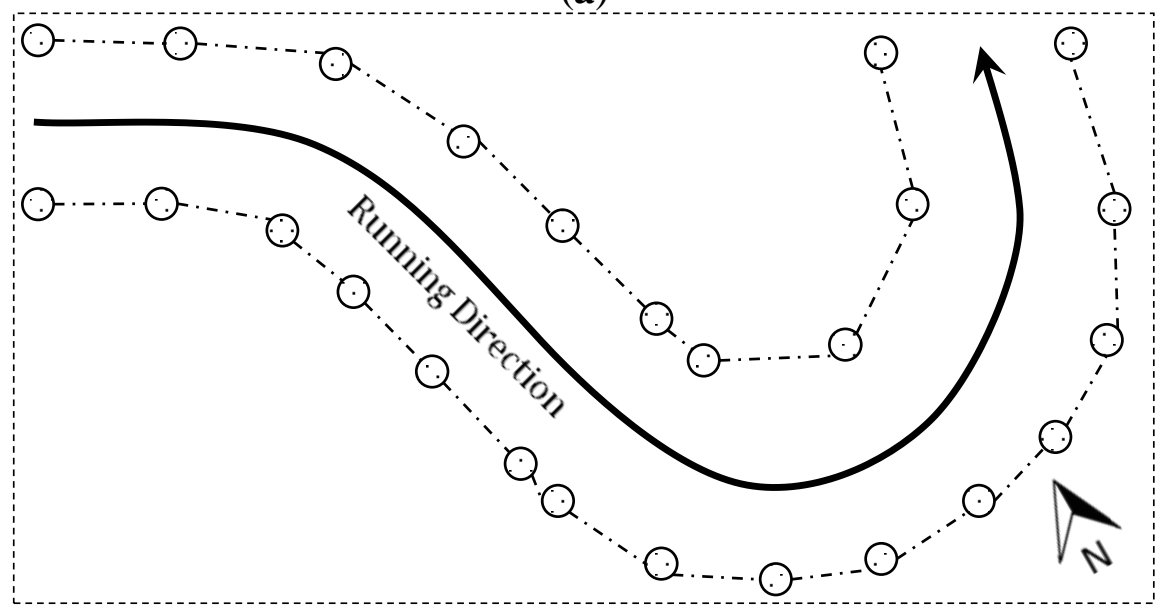

(b)

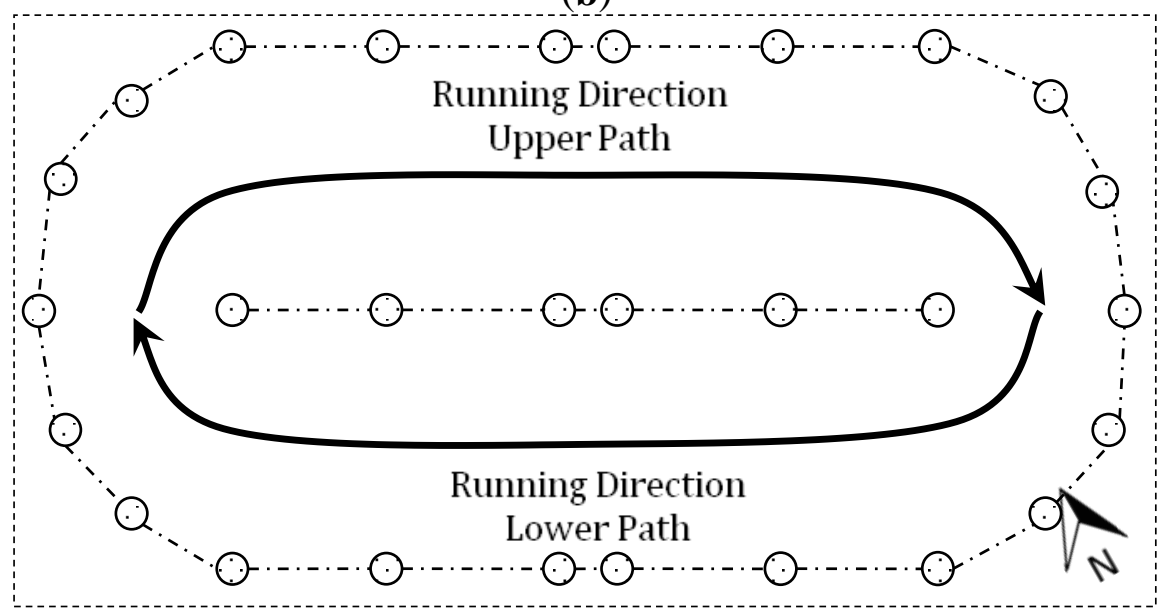

(c)

Figure 16. Three test courses for experiments (a) wide curve; (b) tight curve and (c) U-turn run.

\subsubsection{Accuracy of the Control System}

The accuracy of the navigation system was evaluated by comparing the positioning of the tractor between the autonomous and the farm operator driven methods. The root mean square (RMS) differences in the tractor's position at the $\mathrm{x}$ and $\mathrm{y}$-axes were calculated. For all experiments, the 
tractor was driven at a speed of $0.18 \mathrm{~m} / \mathrm{s}$. The steering angle $(\alpha)$ was also observed during the experiments to evaluate the performance of the steering control system.

\section{Results}

\subsection{Comparison of Path Execution for Manual and Autonomous Runs}

The results show that the navigation system could navigate the tractor to run along the test course without collision with the traffic cones (Figure 17).

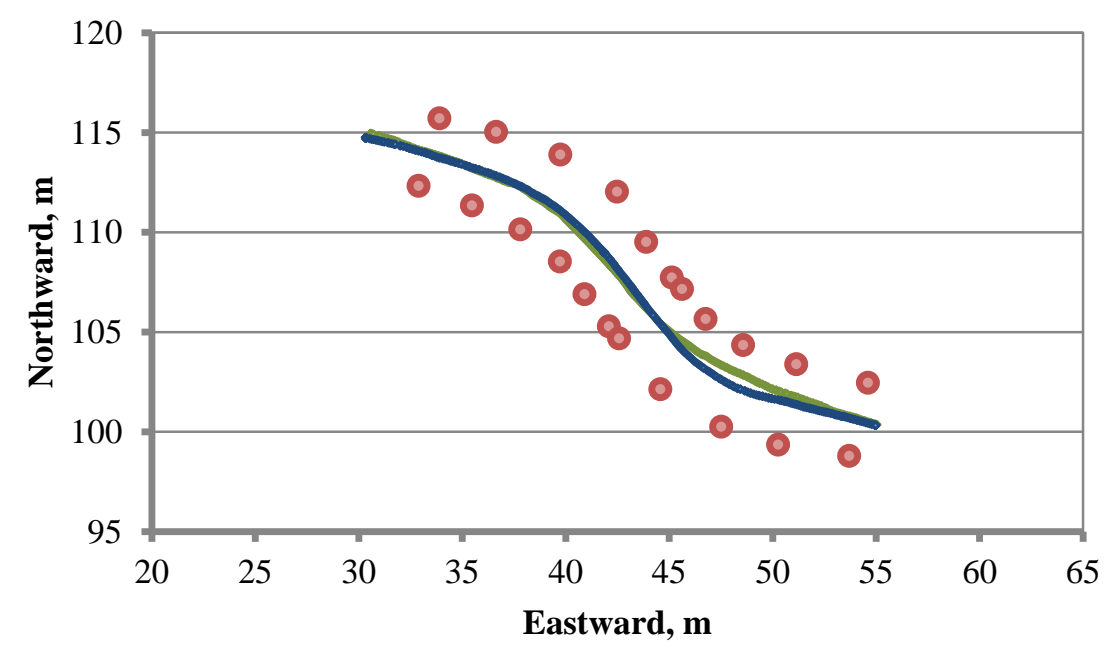

(a)

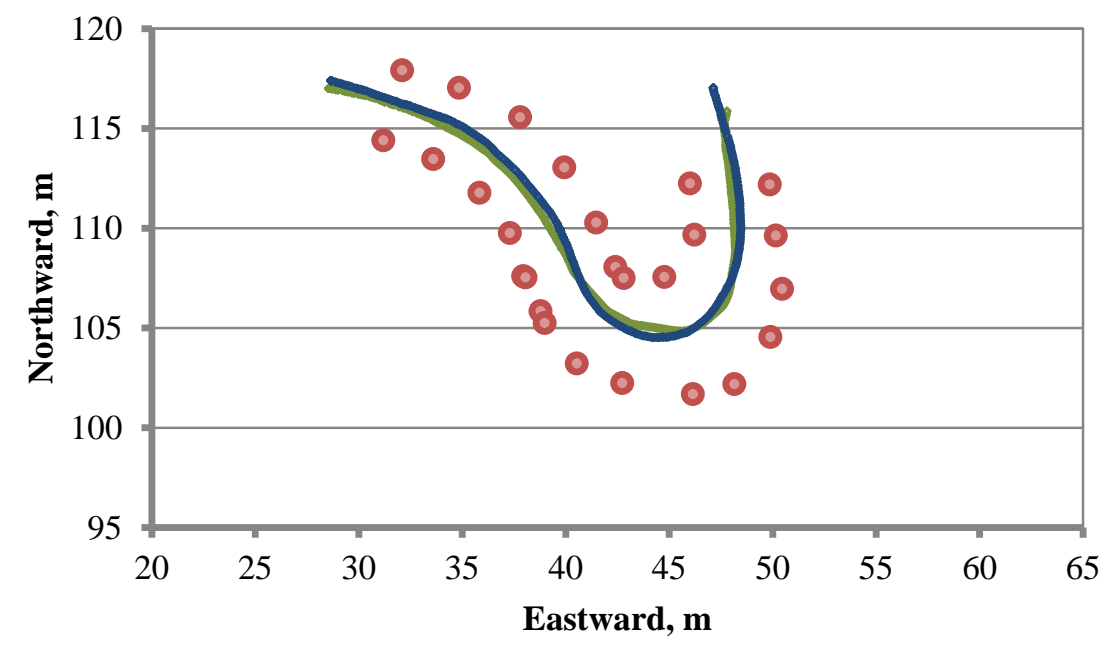

(b)

Figure 17. Cont. 


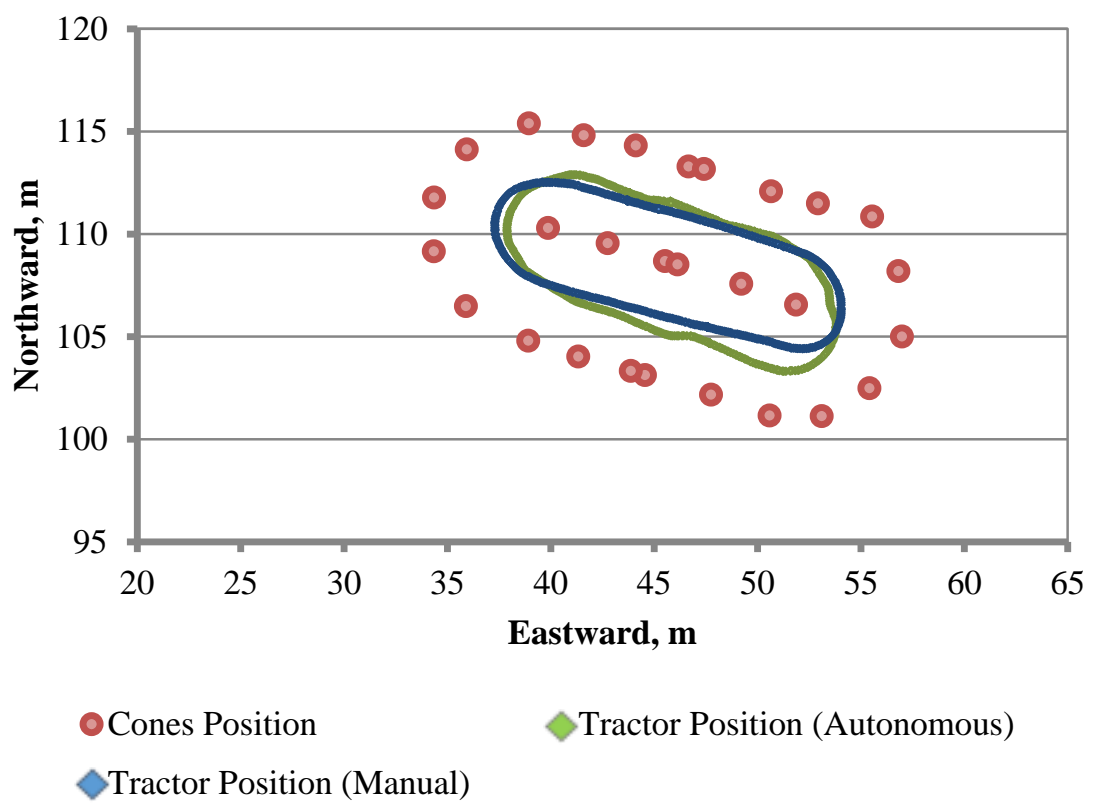

(c)

Figure 17. Comparison of tractor positions when operated autonomously and manually (a) wide curve; (b) tight curve and (c) U-turn run.

In wide curve, the path consisted of a straight path with two wide curves, starting with a wide right turn, then a short straight run before a wide left turn. The RMS differences in position mostly happened at the curve, where the control system tended to drive close to the inner belt of the test course (Figure 17a, 18a). The navigation was performed smoothly using the point-to-go algorithm, and the average RMS difference was $0.264 \mathrm{~m}$ (Table 1).

In tight curve, there were two curves: the path started with a wide curve to the right and then made a near U-turn. The landmarks were recognized continuously while the tractor ran autonomously. However, the navigation system also tended to drive the tractor close to the inner belt of the test course (Figure 17b). The RMS difference in position was higher at the starting point of navigation. Once the LRF detected multiple landmarks and the tractor came closer to the centerline of the path, the RMS difference was decreased. The RMS difference also increased during the turning period (Figure 18b). However, the navigation system provided satisfactory performance while travelling the complete navigation path. The RMS position difference on this course was $0.370 \mathrm{~m}$ (Table 1).

In U-turn run, the experiment started on a straight run before making a U-turn to a straight path and then making a second U-turn to return to the starting point. The results show that the navigation system started to turn faster than the operator, but finished the U-turn slower than the operator. This made the tractor run closer to the inner belt at the beginning of the U-turn and closer to the outer belt near the finish, compared to the manually operated tractor (Figure 17c). The RMS differences increased when making a U-turn and decreased when returning to the straight path (Figure 18c,d). The average RMS difference in this navigation course was $0.542 \mathrm{~m}$ (Table 1). We observed that the proposed navigation system based on the point-to-go calculation using landmarks navigated the tractor with satisfactory accuracy. 


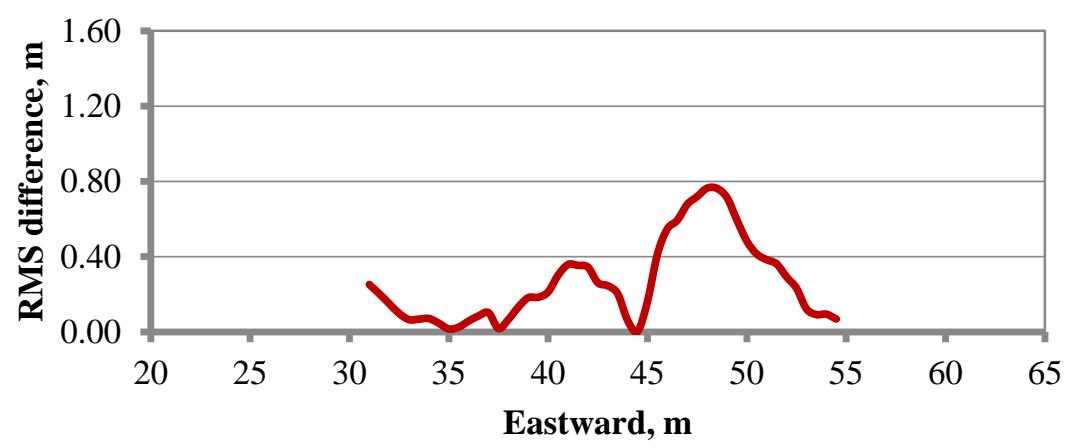

(a)

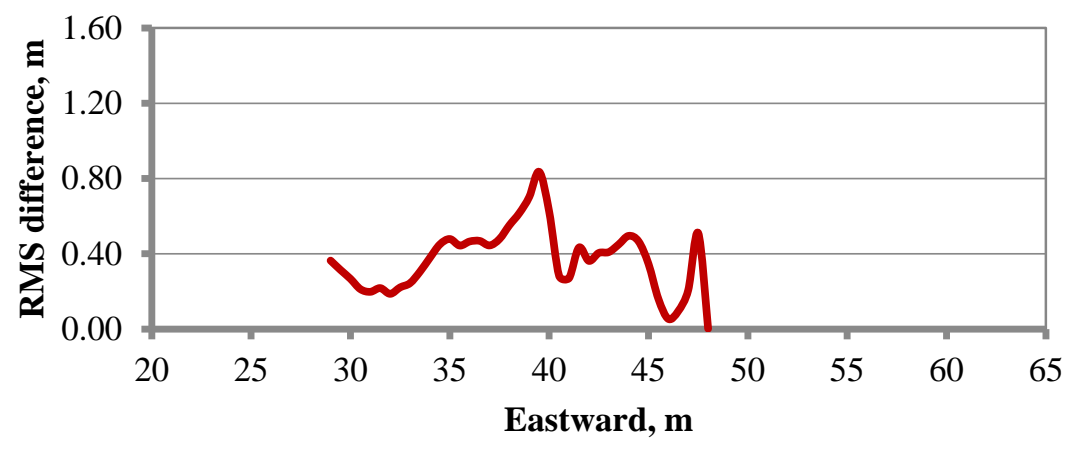

(b)

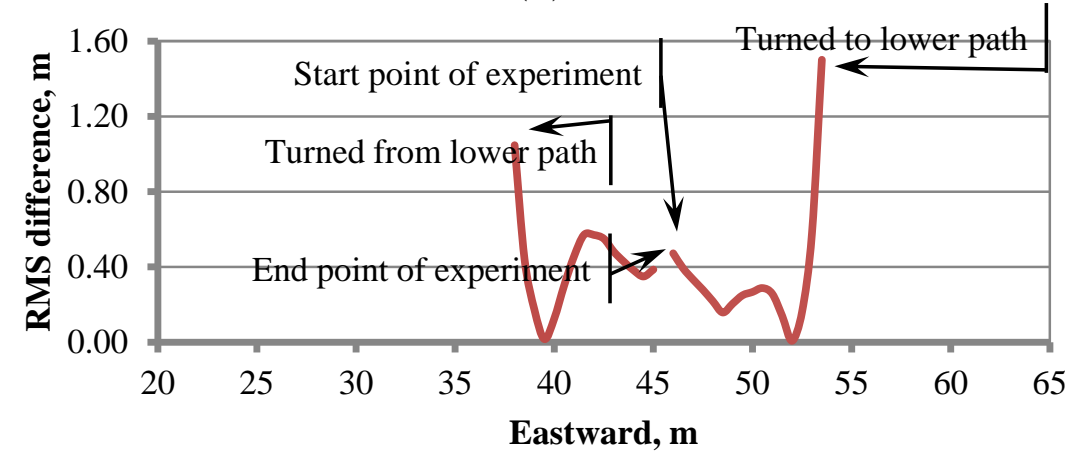

(c)

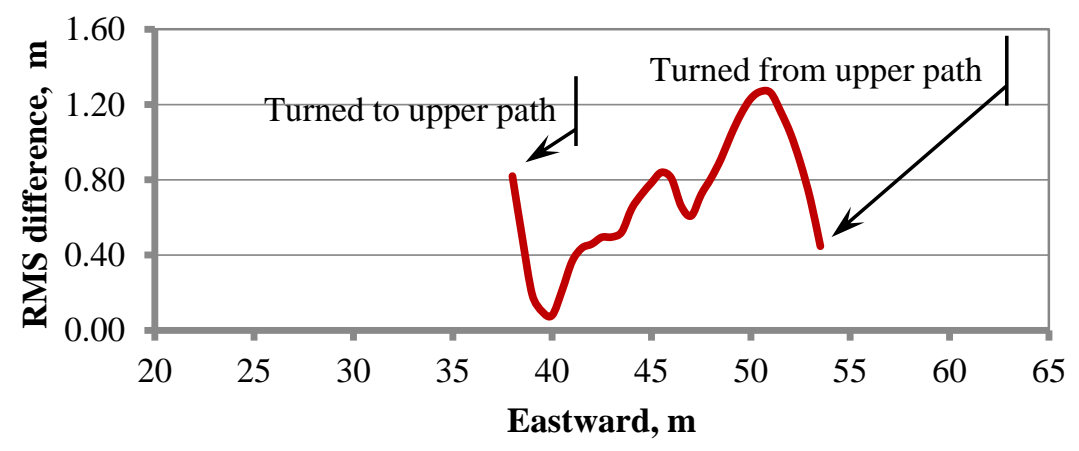

(d)

Figure 18. RMS difference of tractor position when operated autonomously and manually (a) wide curve; (b) tight curve; (c) U-turn run-upper-path and (d) U-turn run-lower path. 
Table 1. Average RMS difference of the tractor's position under autonomous control compared to operator control.

\begin{tabular}{cc}
\hline Test Course & Average RMS Position Difference, m \\
\hline Test course 1 (wide curve) & 0.264 \\
Test course 2 (tight curve) & 0.370 \\
Test course 3 (U-turn run) & 0.542 \\
\hline
\end{tabular}

\subsection{Performance Evaluation of Steering Angle}

The steering angle was observed during the experiments. The measured values were compared with the calculated values (Figure 19a-d). In all the experiments, the measured values had satisfactory accuracy. The only high fluctuations in the steering angle occurred when the LRF detected new landmarks and calculated the next point-to-go. Once the detection was done, the tractor was navigated to the direction of the new point-to-go. Small fluctuations in the measured values were observed when the tractor navigated to the detected targets on the run. However, once the tractor found the new landmarks, the new steering angle was calculated, and then the actuator controlled the steering wheel, resulted in increases in the RMS errors (Figure 20a-d). The RMS errors of the measured steering angle were $3.139^{\circ}, 4.394^{\circ}$ and $5.217^{\circ}$ for the wide curve, tight curve and U-turn runs, respectively (Table 2).

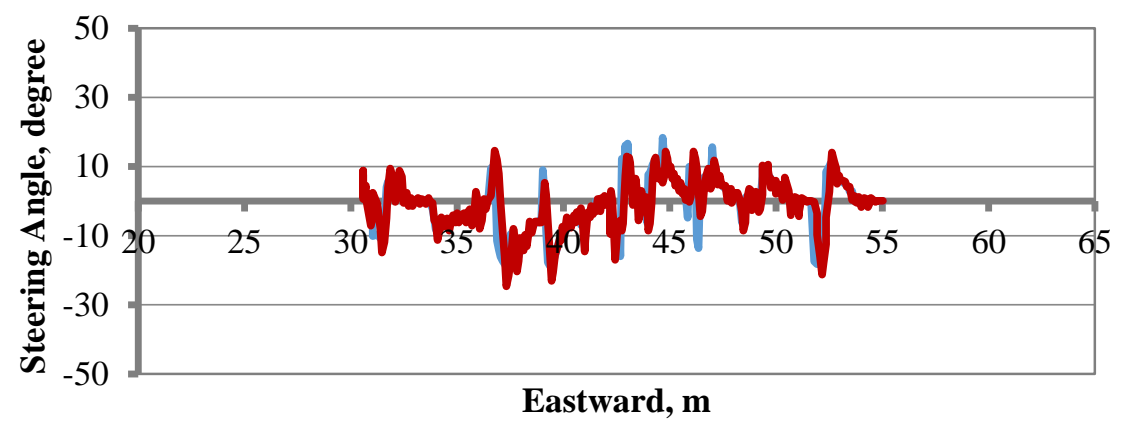

(a)

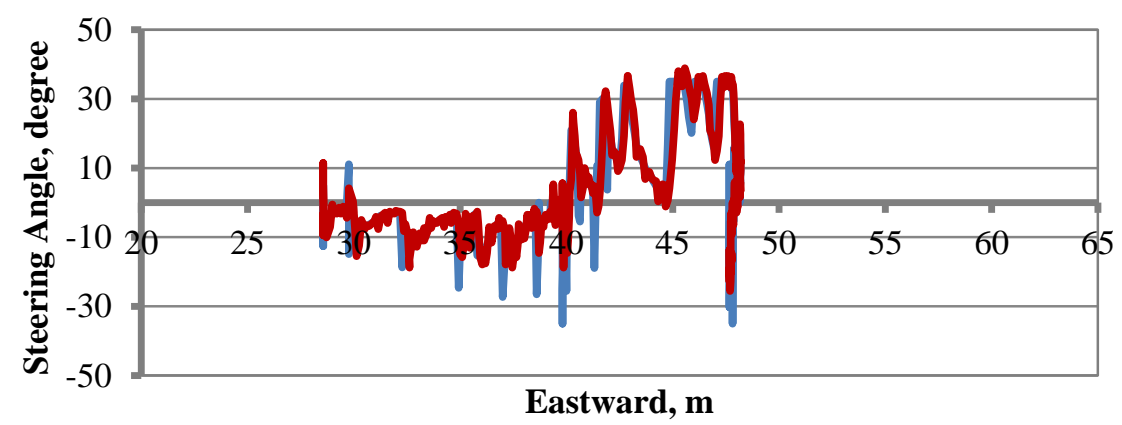

(b)

Figure 19. Cont. 


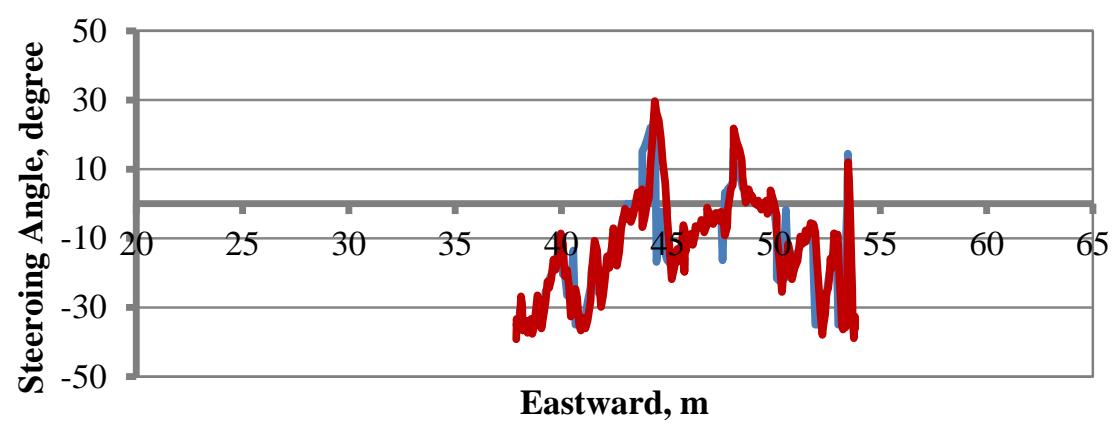

(c)

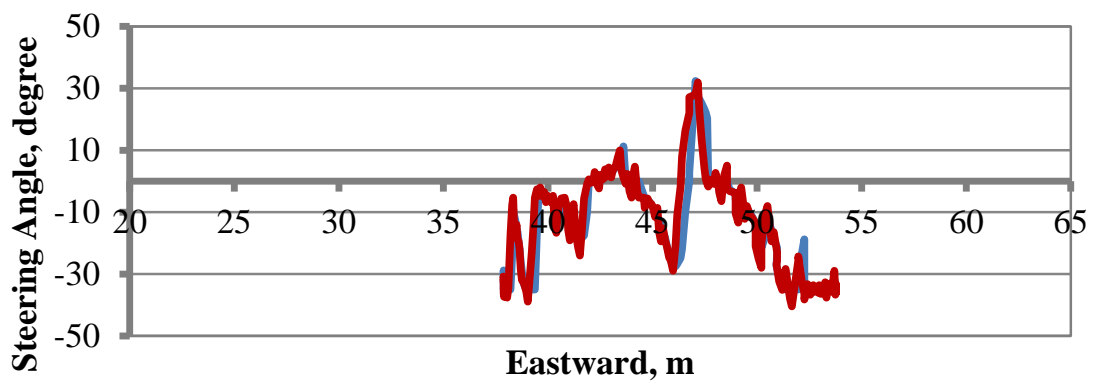

Calculated Steering Angle, degree

Measured Steering Angle, degree

(d)

Figure 19. Tractor's steering angle in experiments (a) wide curve; (b) tight curve; (c) U-turn run-upper-path and (d) U-turn run-lower path.

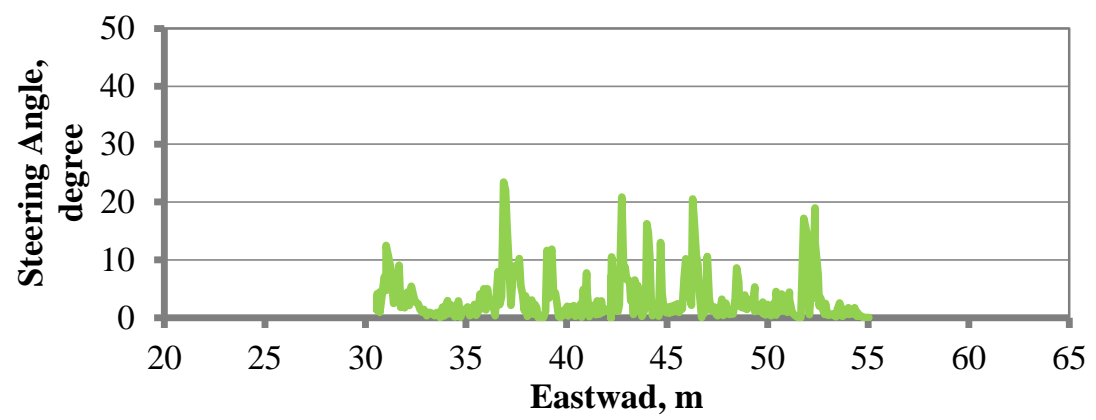

(a)

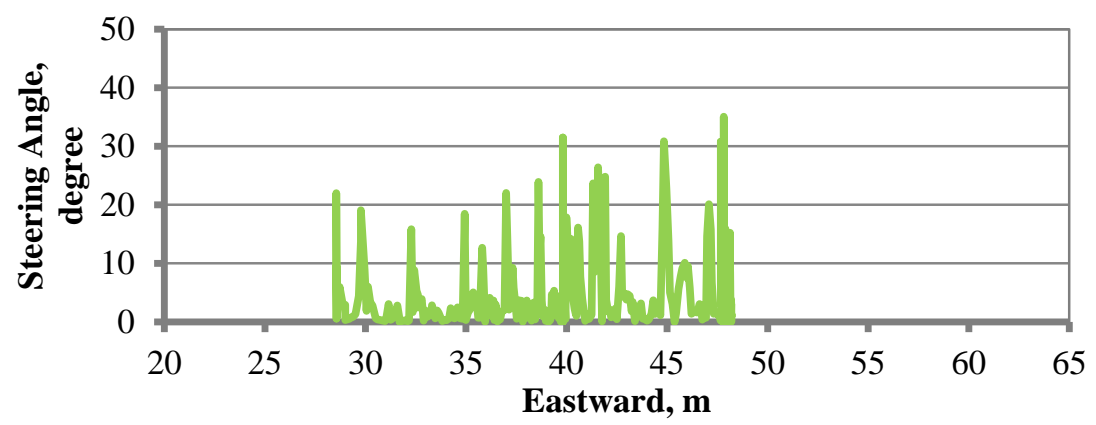

(b)

Figure 20. Cont. 


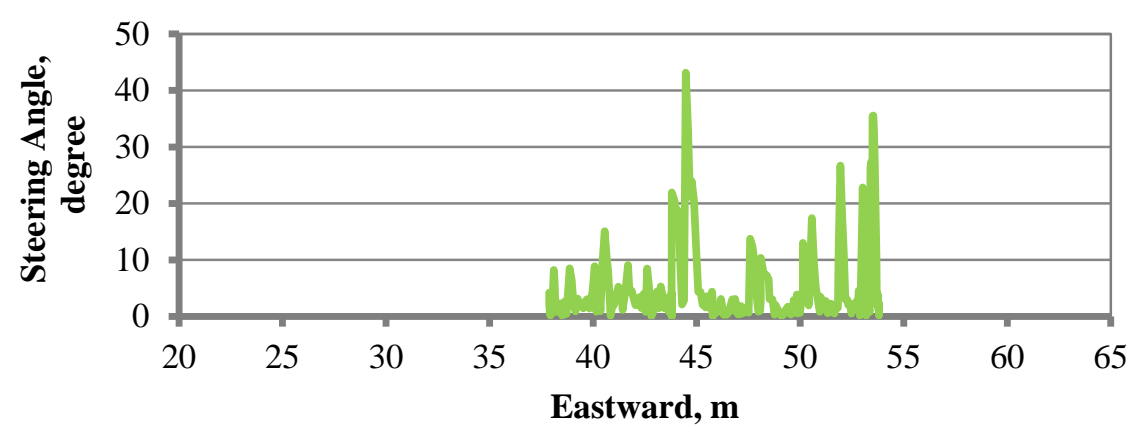

(c)

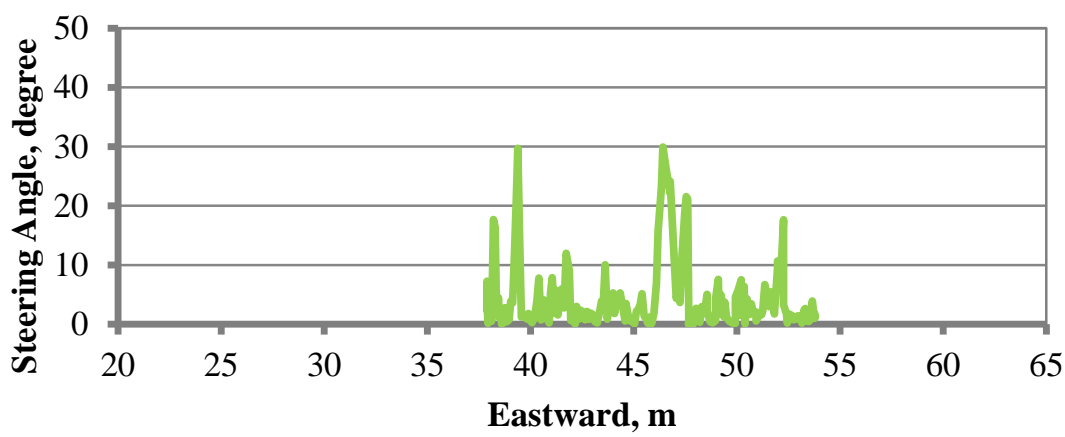

(d)

Figure 20. RMS errors of measured steering angle compared to calculated steering angle (a) wide curve; (b) tight curve; (c) U-turn run-upper-path and (d) U-turn run-lower path.

Table 2. Average RMS difference of the tractor's steering angle measured under autonomous control compared to calculated values.

\begin{tabular}{cc}
\hline Test Course & Average RMS Position Difference, Degree \\
\hline Test course 1 (wide curve) & 3.139 \\
Test course 2 (tight curve) & 4.394 \\
Test course 3 (U-turn run) & 5.217 \\
\hline
\end{tabular}

\section{Discussion}

The navigation system was capable of working where the GPS signal performed poorly and the RTK-fix mode could not be used. Successful navigation was performed on the test courses using landmarks. Performing the experiments on a restricted area shows the performance of the algorithm in a low-noise condition, which resembles a system with a more powerful noise removal algorithm. When the number of landmarks was limited, or no landmarks were detected, the navigation system was controlled, and the tractor was stopped. In both the initial and final conditions of the tractor`s movement, we observed errors. The highest difference in the position of the tractor-trailer navigated autonomously compared with that driven by a human operator was $0.542 \mathrm{~m}$ on a $5 \mathrm{~m}$ width U-turn test course. The higher differences occurred at the curve section of the test path, while on a straight section, the RMS differences decreased. The RMS position differences seem high from a traditional view of theory calculation and experiments following that theory comparison. On the contrary, from the standpoint of how well the autonomous vehicle robot worked compared to a human being, the RMS differences in 
position were acceptable, although there are still gaps to be improved upon. The RMS differences were also higher from the initial position until the tractor was closer to the navigation path. At the end of the travelling course, the error was higher due to the detection of fewer landmarks. For this reason, the point-to-go calculation was shifted from the middle of the navigation path at the end of the course. However, we focused on the entire navigation scheme, which is very important in agricultural operations. Under working conditions, the tractor may not need to operate at the beginning or end of plant rows. Accuracy is needed during the navigation scheme. The control system calculated the first point-to-go from the landmarks at the very beginning of the course and navigated the tractor onto the path. In the case of a plantation with rows of trees or plants, the system could navigate the tractor with enough accuracy to complete the fieldwork, such as in a rubber plantation, a palm oil plantation, or an orchard. The control system could work while plowing, fertilizing, or harvesting plants in the rows. The navigation system could also be used for travelling between plantation areas, where the paths include not only straight sections but also curves or corners. Deploying the navigation systems in plantations, there is a chance of missing the landmarks. For example, during travelling between plots, where the tree arrangement is not in a regular pattern, the use of artificial landmarks could improve the navigation performance.

\section{Conclusions}

A navigation control system was developed for an autonomous tractor using an LRF. The positions of the landmarks were recognized, and the tractor was navigated based on point-to-go calculations. The relative positioning principle was used for determining the steering angle while travelling the courses. The experiment was conducted for wide, tight, and U-turn curves. The control system navigated the tractor on these test courses with accuracies of $0.264,0.370$ and $0.542 \mathrm{~m}$ for the wide, tight, and U-turn curves, respectively, and the average directional errors were $3.139^{\circ}, 4.394^{\circ}$ and $5.217^{\circ}$. This positioning accuracy was satisfactory on the given paths. Because the goal is achieved with satisfactory accuracy, the autonomous navigation system could enable operations in rubber plantations, palm oil plantations and travelling between plantations along straight and curve paths, including- $U$ turns. Further research will be conducted to develop a tractor-trailer system for loading-unloading products at the field level.

\section{Author Contributions}

All authors made substantial contribution to this research. T.T. and T.A. addressed the navigation concept and supervised the research. P.T. developed the navigation algorithms, performed the experiments, analyzed the results and wrote the manuscript. All authors discussed and commented on the manuscript at all stages, interpreted the results, agreed about the conclusions, and further research directions.

\section{Conflicts of Interest}

The authors declare no conflict of interest. 


\section{References}

1. O’Conner, M.; Bell, T.; Elkaim, G.; Parkinson, B. Automatic steering of farm vehicles using GPS. In Proceedings of the Third International Conference on Precision Agriculture, Minneapolis, MN, USA, 23-26 June 1996; pp. 767-778.

2. O’Connor, M.; Elkaim, G.H.; Parkinson, B.W. Carrier-phase DGPS for closed-loop control of farm and construction vehicles. J. Inst. Navig. 1996, 43, 167-178.

3. Stombaugh, T.S.; Bensen, E.R.; Hummel, J.W. Guidance of agricultural vehicles at high field speeds. Trans. ASABE 1999, 42, 537-544.

4. Van Zuydam, R.P. A driver's steering aid for an agricultural implement, based on an electronic map and real time kinematic DGPS. Comput. Electron. Agric. 1999, 24, 153-163.

5. Bell, T. Automatic tractor guidance using carrier-phase differential GPS. Comput. Electron. Agric. 2000, 25, 53-66.

6. Nagasaka, Y.; Umeda, N.; Kanetai, Y.; Taniwaki, K.; Sasaki, Y. Autonomous guidance for rice transplanting using global positioning and gyroscopes. Comput. Electron. Agric. 2004, 43, 223-234.

7. Casciati, F.; Domanesshi, M.; Faravelli, L. Design and implementation of a pointer system controller. Nonlinear Dyn. 2004, 36, 203-215.

8. Casciati, F.; Fuggini, C. Monitoring a steel building using GPS sensors. Smart Struct. Syst. 2011, 7, 349-363.

9. Wu, L.; Casciati, F. Local positioning systems versus structural monitoring: A review. Struct. Control Health Monit. 2014, 21, 1209-1221.

10. Barawid, O.C., Jr.; Mizushima, A.; Ishii, K.; Noguchi, N. Development of an autonomous navigation system using a two-dimensional laser scanner in an orchard application. Biosyst. Eng. 2007, 96, 139-149.

11. Ahamed, T.; Tian, L.; Takigawa, T.; Zhang, Y. Development of auto-hitching navigation system for farm implements using laser range finder. Trans. ASABE 2009, 52, 1793-1803.

12. Ahamed, T.; Takigawa, T.; Koike, M.; Honma, T.; Hasegawa, H.; Zhang, Q. Navigation using a laser range finder for autonomous tractor (part 1)-Positioning of implement. J. JSAM 2006, 68, $68-77$.

13. Ahamed, T.; Takigawa, T.; Koike, M.; Honma, T.; Hasegawa, H.; Zhang, Q. Navigation using a laser range finder for autonomous tractor (part 2) - Navigation for approach composed of multiple paths. J. JSAM 2006, 68, 78-86.

14. Takigawa, T.; Sutiarso, L.; Koike, M.; Kurosaki, H.; Hasegawa, H. Trajectory control and its application to approach a target: Part I. Development of trajectory control algorithms for an autonomous vehicle. Trans. ASAE 2002, 45, 1191-1197.

15. Sutiarso, L.; Kurosaki, H.; Takigawa, T.; Koike, M.; Yukumoto, O.; Hasegawa, H. Trajectory control and its application to approach a target: Part II. Target approach experiments. Trans. ASAE 2002, 45, 1199-1205.

16. Hamner, B.; Bergerman, M.; Singh, S. Autonomous orchard vehicles for specialty crops production. In ASABE Annual International Meeting; ASABE: Louisville, KY, USA, 2011. 
17. Zhang, J.; Chambers, A.; Maeta, S.; Bergerman, M.; Singh, S. 3D perception for accurate row following: Methodology and results. In Proceedings of the IEEE/RSJ International Conference on Intelligent Robots and Systems (IROS), Tokyo, Japan, 3-7 November 2013.

18. Subramanian, V.; Burks, T.F.; Arroyo, A.A. Development of machine vision and laser radar based autonomous vehicle guidance systems for citrus grove navigation. Comput. Electron. Agric. 2006, 53, 130-143.

19. Choi, J.; Yin, X.; Yang, L.; Noguchi, N. Development of a laser scanner-based navigation system for a combine harvester. Eng. Agric. Environ. Food 2014, 7, 7-13.

20. Kurashiki, K.; Fukao, T.; Nagata, J.; Ichiyama, K.; Kamiya, T.; Murakami, N. Orchard travelling ugv using a laser range finder based localization and inverse optimal control. J. Robot. Soc. Jpn. 2014, 30, 428-435.

21. Thanpattranon, P.; Takigawa, T. An application of laser range finder for tractor-trailer navigation in mountainous area, experiment on artifact pathway. In Proceedings of the 69th Japanese Society of Agricultural Machinery Annual Meeting, Ehime, Japan, 14-15 September 2010; pp. 70-71.

22. Thanpattranon, P.; Takigawa, T. Tractor steering control using laser range finder. In Proceedings of the 70th Japanese Society of Agricultural Machinery Annual Meeting, Aomori, Japan, 27-28 September 2011; pp. 244-245.

(C) 2015 by the authors; licensee MDPI, Basel, Switzerland. This article is an open access article distributed under the terms and conditions of the Creative Commons Attribution license (http://creativecommons.org/licenses/by/4.0/). 